\title{
Positive Solution to a Singular $p$-Laplacian BVP with Sign-Changing Nonlinearity Involving Derivative on Time Scales
}

\author{
You-Hui Su, ${ }^{1}$ Subei Li, ${ }^{1}$ and Can-Yun Huang ${ }^{2}$ \\ ${ }^{1}$ School of Mathematics and Physics, Xuzhou Institute of Technology, Xuzhou, Jiangsu 221008, China \\ ${ }^{2}$ Department of Applied Mathematics, Lanzhou University of Technology, Lanzhou 730050, China
}

Correspondence should be addressed to You-Hui Su, suyouhui@xzit.edu.cn

Received 3 January 2009; Revised 4 March 2009; Accepted 24 March 2009

Recommended by Alberto Cabada

Let $\mathbb{T}$ be a time scale such that $0, T \in \mathbb{T}$. By using a monotone iterative method, we present some existence criteria for positive solution of a multiple point general Dirichlet-Robin BVP on time scales with the singular sign-changing nonlinearity. These results are even new for the corresponding differential $(\mathbb{T}=\mathbb{R})$ and difference equation $(\mathbb{T}=\mathbb{Z})$ as well as in general time scales setting. As an application, an example is given to illustrate the results. The interesting point here is that the sign-changing nonlinear term is involved with the first-order derivative explicitly, and the singularity may occur at $u=0, t=0$, and $t=T$.

Copyright (C) 2009 You-Hui Su et al. This is an open access article distributed under the Creative Commons Attribution License, which permits unrestricted use, distribution, and reproduction in any medium, provided the original work is properly cited.

\section{Introduction}

Initiated by Hilger in his Ph.D. thesis [1] in 1988, the theory of time scales has been improved greatly ever since. In particular, considerable works have been made in the existence problems of solutions of dynamic systems on time scales, for details, see [2-12] and the references therein. The reason for that lies in two aspects. On one hand, the time scales approach not only unifies differential and difference equations, but also solves other problems that are a mix of stop-start and continuous behavior. On the other hand, the time scales calculus has a tremendous potential for application, for example, Hoffacker et al. have used the theory to model how students suffering from the eating disorder bulimia are influenced by their college friends. With the theory on time scales, they can model how the number of sufferers changes during the continuous college term as well as during long breaks [13]. Moreover, the theory is widely applied to the research of biology, heat transfer, stock market, wound healing and epidemic models [3, 13-16], and so forth. 
Here and hereafter, we denote $\varphi_{p}(u)$ as $p$-Laplacian operator, that is, $\varphi_{p}(u)=|u|^{p-2} u$ for $p>1$ and $\left(\varphi_{p}\right)^{-1}=\varphi_{q}$, where $1 / p+1 / q=1$. We make the blanket assumption that $0, T$ are points in $\mathbb{T}$, by an interval $(0, T)_{\mathbb{T}}$ we always mean $(0, T) \cap \mathbb{T}$. Other types of interval are defined similarly.

In [17], Su et al. concerned with the $m$-point singular $p$-Laplacian boundary value problem of the form

$$
\begin{gathered}
\left(\varphi_{p}\left(u^{\Delta}(t)\right)\right)^{\nabla}+q(t) f(t, u(t))=0, \quad t \in(0, T)_{\mathbb{T}} \\
u(0)=0, \quad u(T)-\sum_{i=1}^{m-2} \psi_{i}\left(u\left(\xi_{i}\right)\right)=0
\end{gathered}
$$

and obtained some existence criteria for positive solutions of boundary value problem (1.1). Yet, the singularity of nonlinear term of boundary value problem (1.1) is only occur at $u=0$. As a result, they failed to further provide comprehensible results of the singularity that may occur at $u=0, t=0$, or $t=T$. Now, it is natural to consider the existence of positive solutions of $p$-Laplacian dynamic equations with the singularity that may occur at $u=0, t=0$, and $t=T$ in all respects.

For the existence problems of positive solutions of singular $p$-Laplacian boundary value problem with sign changing nonlinearity on time scales, some authors have obtained a few results, for details, see [17-20] and the references therein. It is also noted that the above-mentioned references [17-20] only considered the existence of positive solutions of boundary value problems with nonlinear terms that are not involved with first-order derivative explicitly. Naturally, it is quite necessary to consider that the existence of positive solutions for $p$-Laplacian dynamic equations with the nonlinear term is involved with the first-order derivative explicitly.

Motivated by the above-mentioned ideas, we all-sidedly consider the multiple point singular $p$-Laplacian boundary value problem on time scales of the form

$$
\begin{gathered}
\left(\varphi_{p}\left(u^{\Delta}(t)\right)\right)^{\nabla}+q(t) f\left(t, u(t), u^{\Delta}(t)\right)=0, \quad t \in(0, T)_{\mathbb{T}}, \\
u(0)=0, \quad \sum_{j=1}^{m_{1}} \phi_{j}\left(u\left(\xi_{j}^{\prime}\right)\right)-\sum_{i=1}^{m_{2}} \psi_{i}\left(u^{\Delta}\left(\xi_{i}\right)\right)=0, \quad m_{1}, m_{2} \in\{1,2, \ldots\},
\end{gathered}
$$

where $\varphi_{p}(u)=|u|^{p-2} u$ for $p>1, \phi_{j}, \psi_{i}: \mathbb{R} \rightarrow \mathbb{R}$ are continuous, nondecreasing and $\phi_{j}, \psi_{i}$ may be nonlinear, $0<\xi_{1}^{\prime}<\xi_{2}^{\prime}<\cdots<\xi_{m_{1}-1}^{\prime}<\xi_{m_{1}}^{\prime}=T$, and $0<\xi_{1}<\xi_{2}<\cdots<\xi_{m_{2}} \leq T$. The singularity may occur at $u=0, t=0$, or $t=T$, and the nonlinearity is allowed to change sign and is involved with the first-order derivative explicitly. In particular, the boundary condition (1.3) includes the Dirichlet boundary condition and Robin boundary condition. By applying a monotone iterative method, we obtain some new existence criteria for positive solutions of the boundary value problem (1.2) and (1.3). Our results are even new for the corresponding differential $(\mathbb{T}=\mathbb{R})$ and difference equations $(\mathbb{T}=\mathbb{Z})$ as well as in general time scales setting. It has been well known that a second-order dynamic derivative does not approximate a second-order derivative nor a conventional difference; see [21-23]. Thus, it would be interesting that the mathematical results obtained in our article can be conveniently extended for differential or difference equations. 
As an application, an example is given to illustrate these results. In particular, our results improve and generalize some known works of Agarwal et al. [24], O'Regan [25] ( $p=$ $2)$, Lü et al. [26, 27] when $\mathbb{T}=\mathbb{R}$; extend and include the results of Lü et al. [28] in the case of $\mathbb{T}=\mathbb{R}$; if $f\left(t, u, u^{\Delta}\right)=f(t, u)$, then the works of $[17,19]$ are only the special cases of our results.

For the convenience of statements, we present some basic definitions and lemmas concerning the calculus on time scales that one needs to read this paper, which can be found in $[2,3]$. One of another excellent sources on dynamical systems on time scales is from the book in [29].

A time scale $\mathbb{T}$ is a nonempty closed subset of $\mathbb{R}$. It follows that the jump operators $\sigma, \rho: \mathbb{T} \rightarrow \mathbb{T}$ defined by $\sigma(t)=\inf \{\tau \in \mathbb{T}: \tau>t\}$ and $\rho(t)=\sup \{\tau \in \mathbb{T}: \tau<t\}$ (supplemented by $\inf \emptyset:=\sup \mathbb{T}$ and $\sup \emptyset:=\inf \mathbb{T})$ are well defined. The point $t \in \mathbb{T}$ is left dense, left scattered, right dense, right scattered if $\rho(t)=t, \rho(t)<t, \sigma(t)=t, \sigma(t)>t$, respectively. If $\mathbb{T}$ has a right-scattered minimum $m$, define $\mathbb{T}_{\kappa}=\mathbb{T}-\{m\}$; otherwise, set $\mathbb{T}_{\kappa}=\mathbb{T}$. If $\mathbb{T}$ has a leftscattered maximum $M$, define $\mathbb{T}^{\kappa}=\mathbb{T}-\{M\}$; otherwise, set $\mathbb{T}^{\kappa}=\mathbb{T}$. The forward graininess is $\mu(t):=\sigma(t)-t$. Similarly, the backward graininess is $v(t):=t-\rho(t)$.

A function $f: \mathbb{T} \rightarrow \mathbb{R}$ is ld-continuous provided that it is continuous at left-dense points in $\mathbb{T}$ and its right-sided limit exists (finite) at right-dense points in $\mathbb{T}$. It is known [3] that if $f$ is ld-continuous, then there is a function $F(t)$ such that $F^{\nabla}(t)=f(t)$. In this case, we define $\int_{a}^{b} f(\tau) \nabla \tau=F(b)-F(a)$.

Throughout this paper, it is assumed that

(H1) $f(t, x, y):(0, T)_{\mathbb{T}} \times(0, \infty) \times(-\infty, \infty) \rightarrow \mathbb{R}$ is continuous;

(H2) $q(t) \in C\left((0, T)_{\mathbb{T}},(0, \infty)\right)$ and $\int_{0}^{T} q(t) \nabla t<\infty$;

(H3) $\phi_{j}, \psi_{i}: \mathbb{R} \rightarrow \mathbb{R}$ are continuous and nondecreasing, where $j=1,2, \ldots, m_{1}$ and $i=$ $1,2, \ldots, m_{2}$.

\section{Existence Results}

Let $E=C\left([0, T]_{\mathbb{T}}, \mathbb{R}\right) \cap C^{\Delta}\left((0, T]_{\mathbb{T}^{\kappa}}, \mathbb{R}\right)$, and define the norm with

$$
\|u\|=\max \left\{\sup _{t \in[0, T]_{\mathbb{T}}}|u(t)|, \sup _{t \in[0, T]_{\mathbb{T}^{\kappa}}}\left|u^{\Delta}(t)\right|\right\},
$$

then $E$ is a Banach space.

To demonstrate existence of positive solutions to problem (1.2) and (1.3), we firstly approximate the singular problem by means of a sequence of nonsingular problems, and by using the lower and upper solutions for nonsingular problem together with Schauders fixed point theorem, and then we establish the existence of solutions to each approximating problem. We remark here that the singularity of the following results occurs at $u=0, t=0$, or $t=T$. 
Now we state and prove our main result.

Theorem 2.1. Let $n_{0} \in\{1,2, \ldots\}$ be fixed. Assume that (H1)-(H3) hold and the following conditions are satisfied:

(A1) for each $n \in\left\{n_{0}, n_{0}+1, \ldots\right\} \equiv \mathbb{N}_{1}$, there exists a constant sequence $\rho_{n}$ such that $\left\{\rho_{n}\right\}$ is a strictly monotone decreasing sequence with $\lim _{n \rightarrow \infty} \rho_{n}=0$, and $q(t) f\left(t, \rho_{n}, 0\right) \geq 0$ for $t \in\left[1 / 2^{n+1}, T-\left(1 / 2^{n+1}\right)\right]_{\mathbb{T}} ;$

(A2) there exists a function $\alpha(t) \in C[0, T]_{\mathbb{T}} \cap C^{\Delta}(0, T]_{\mathbb{T}}, \varphi_{p}\left(\alpha^{\Delta}(t)\right) \in C^{\nabla}(0, T)_{\mathbb{T}}$ such that $\alpha(0)=0, \alpha(t)>0$ on $(0, T]_{\mathbb{T}}, \sum_{j=1}^{m_{1}} \phi_{j}\left(\alpha\left(\xi_{j}^{\prime}\right)\right)-\sum_{i=1}^{m_{2}} \psi_{i}\left(\alpha^{\Delta}\left(\xi_{i}\right)\right) \leq 0$ together with $-\left(\varphi_{p}\left(\alpha^{\Delta}(t)\right)\right)^{\nabla} \leq q(t) f\left(t, \alpha(t), \alpha^{\Delta}(t)\right)$ for $t \in(0, T)_{\mathbb{T}} ;$

(A3) there exists a function $\beta(t) \in C[0, T]_{\mathbb{T}} \cap C^{\Delta}(0, T]_{\mathbb{T}^{\kappa}}, \varphi_{p}\left(\beta^{\Delta}(t)\right) \in C^{\nabla}(0, T)_{\mathbb{T}}$ such that $\beta(t) \geq \alpha(t)$ and $\beta(t) \geq \rho_{n_{0}}$ for $t \in[0, T]_{\mathbb{T}}, \sum_{j=1}^{m_{1}} \phi_{j}\left(\beta\left(\xi_{j}^{\prime}\right)\right)-\sum_{i=1}^{m_{2}} \psi_{i}\left(\beta^{\Delta}\left(\xi_{i}\right)\right)>0$ and $-\left(\varphi_{p}\left(\beta^{\Delta}(t)\right)\right)^{\nabla} \geq q(t) f\left(t, \beta(t), \beta^{\Delta}(t)\right)$ for $t \in\left[1 / 2^{n_{0}+1}, T-\left(1 / 2^{n_{0}+1}\right)\right]_{\mathbb{T}}$, with

$$
\begin{aligned}
& -\left(\varphi_{p}\left(\beta^{\Delta}(t)\right)\right)^{\nabla} \geq q(t) f\left(\frac{1}{2^{n_{0}+1}}, \beta(t), \beta^{\Delta}(t)\right) \text { for } t \in\left(0, \frac{1}{2^{n_{0}+1}}\right)_{\mathbb{T}}, \\
& -\left(\varphi_{p}\left(\beta^{\Delta}(t)\right)\right)^{\nabla} \geq q(t) f\left(T-\frac{1}{2^{n_{0}+1}}, \beta(t), \beta^{\Delta}(t)\right) \text { for } t \in\left(T-\frac{1}{2^{n_{0}+1}}, T\right)_{\mathbb{T}}
\end{aligned}
$$

Then the boundary value problem (1.2) and (1.3) has a positive solution $u(t) \in C[0, T]_{\mathbb{T}} \cap C^{\Delta}(0, T]_{\mathbb{T}^{x}}$, $\varphi_{p}\left(u^{\Delta}(t)\right) \in C^{\nabla}(0, T)_{\mathbb{T}}$, with $u(t) \geq \alpha(t)$ for $t \in[0, T]_{\mathbb{T}}$.

Proof. Let $\xi=\min \left\{\xi_{1}, \xi_{1}^{\prime}\right\}$. Without loss of generality, fix $n \in \mathbb{N}_{1}$, we suppose that $\min _{t \in[\xi, T]_{\mathbb{T}}} \alpha(t) \geq \rho_{n}$, let $t_{n} \in(0, \xi)_{\mathbb{T}}$ be such that

$$
\alpha\left(t_{n}\right)=\rho_{n}, \quad \alpha(t) \leq \rho_{n} \quad \text { for } t \in\left[0, t_{n}\right]_{\mathbb{T}} .
$$

Let

$$
\alpha_{n}(t)=\left\{\begin{array}{ll}
\rho_{n}, & \text { if } t \in\left[0, t_{n}\right]_{\mathbb{T}}, \\
\alpha(t), & \text { if } t \in\left[t_{n}, T\right]_{\mathbb{T}},
\end{array} \quad \text { here } \alpha\left(t_{n}\right)=\rho_{n}\right.
$$

Assume that $e_{n}=\left[1 / 2^{n+1}, T-\left(1 / 2^{n+1}\right)\right]_{\mathbb{T}}$,

$$
\begin{aligned}
& \omega_{n}(t)= \begin{cases}\max \left\{\frac{1}{2^{n+1}}, t\right\}, & \text { for } t \in\left[0, T-\frac{1}{2^{n+1}}\right]_{\mathbb{T}}{ }^{\prime} \\
\min \left\{T-\frac{1}{2^{n+1}}, t\right\}, & \text { for } t \in\left[T-\frac{1}{2^{n+1}}, T\right]_{\mathbb{T}}{ }^{\prime}\end{cases} \\
& f_{n}(t, x, y)=\max \left\{f(t, x, y), f\left(\omega_{n}(t), x, y\right)\right\} \text {. }
\end{aligned}
$$

We define a sequence $h_{n_{0}}(t, x, y)=f_{n_{0}}(t, x, y)$ and

$$
h_{n}(t, x, y)=\min \left\{f_{n_{0}}(t, x, y), \ldots, f_{n}(t, x, y)\right\}, \quad n=n_{0}+1, n_{0}+2, \ldots
$$


Then

$$
\begin{array}{r}
f(t, x, y) \leq \cdots \leq h_{n+1}(t, x, y) \leq h_{n}(t, x, y) \leq \cdots \leq h_{n_{0}}(t, x, y), \\
\text { for }(t, x, y) \in(0, T)_{\mathbb{T}} \times(0, \infty) \times(-\infty,+\infty), \\
h_{n}(t, x, y)=f(t, x, y), \quad \text { for }(t, x, y) \in e_{n} \times(0, \infty) \times(-\infty, \infty) .
\end{array}
$$

Consider the boundary value problem

$$
\begin{gathered}
\left(\varphi_{p}\left(u^{\Delta}(t)\right)\right)^{\nabla}+q(t) h_{n_{0}}^{*}\left(t, u(t), u^{\Delta}(t)\right)=0, \quad t \in(0, T)_{\mathbb{T}} \\
u(0)=\rho_{n_{0}}, \quad \sum_{j=1}^{m_{1}} \phi_{j}^{*}\left(u\left(\xi_{j}^{\prime}\right)\right)-\sum_{i=1}^{m_{2}} \psi_{i}^{*}\left(u^{\Delta}\left(\xi_{i}\right)\right)=\rho_{n_{0}}
\end{gathered}
$$

where

$$
\begin{aligned}
& h_{n_{0}}^{*}\left(t, u(t), u^{\Delta}(t)\right)= \begin{cases}h_{n_{0}}\left(t, \alpha_{n_{0}}(t), \alpha_{n_{0}}^{\Delta}(t)\right)+r\left(\alpha_{n_{0}}(t)-u(t)\right), & u(t) \leq \alpha_{n_{0}}(t), \\
h_{n_{0}}\left(t, u(t), u^{\Delta}(t)\right), & \alpha_{n_{0}}(t) \leq u(t) \leq \beta(t), \\
h_{n_{0}}\left(t, \beta(t), \beta^{\Delta}(t)\right)+r(\beta(t)-u(u)), & u(t) \geq \beta(t),\end{cases} \\
& \phi_{j}^{*}\left(z_{j}^{\prime}\right)= \begin{cases}\phi_{j}\left(\alpha\left(\xi_{j}^{\prime}\right)\right), & z_{j}^{\prime} \leq \alpha_{n_{0}}\left(\xi_{j}^{\prime}\right)=\alpha\left(\xi_{j}^{\prime}\right), \\
\phi_{j}\left(z_{j}^{\prime}\right), & \alpha_{n_{0}}\left(\xi_{j}^{\prime}\right) \leq z_{j}^{\prime} \leq \beta\left(\xi_{j}^{\prime}\right), \quad j=1, \ldots, m_{1}-1, \\
\phi_{j}\left(\beta\left(\xi_{j}^{\prime}\right)\right), & z_{j}^{\prime} \geq \beta\left(\xi_{j}^{\prime}\right),\end{cases} \\
& \psi_{i}^{*}\left(z_{i}\right)= \begin{cases}\psi_{i}\left(\alpha^{\Delta}\left(\xi_{i}\right)\right), & z_{i} \leq \alpha_{n_{0}}^{\Delta}\left(\xi_{i}\right)=\alpha^{\Delta}\left(\xi_{i}\right) \\
\psi_{i}\left(z_{i}\right), & \alpha_{n_{0}}^{\Delta}\left(\xi_{i}\right) \leq z_{i} \leq \beta^{\Delta}\left(\xi_{i}\right), \quad i=1, \ldots, m_{2} \\
\psi_{i}\left(\beta^{\Delta}\left(\xi_{i}\right)\right), & z_{i} \geq \beta^{\Delta}\left(\xi_{i}\right)\end{cases}
\end{aligned}
$$

and $r(u): \mathbb{R} \rightarrow[-1,1]$ is the radial retraction function defined by

$$
r(u)= \begin{cases}u, & |u| \leq 1 \\ \frac{u}{|u|}, & |u|>1\end{cases}
$$

Assume that

$$
C_{0}[0, T]_{\mathbb{T}}=\left\{u \in C[0, T]_{\mathbb{T}}: u(0)=0\right\}, \quad C_{\rho_{n_{0}}}^{\Delta}[0, T]_{\mathbb{T}^{\kappa}}=\left\{u \in C^{\Delta}[0, T]_{\mathbb{T}^{\kappa}}: u(0)=\rho_{n_{0}}\right\}
$$


We define the mappings $L_{p}, F: C_{\rho_{n_{0}}}^{\Delta}[0, T]_{\mathbb{T}^{\kappa}} \rightarrow C_{0}[0, T]_{\mathbb{T}} \times \mathbb{R}$ to be such that

$$
\begin{aligned}
L_{p} u(t) & =\left(\varphi_{p}\left(u^{\Delta}(t)\right)-\varphi_{p}\left(u^{\Delta}(0)\right), \sum_{j=1}^{m_{1}} \phi_{j}^{*}\left(u\left(\xi_{j}^{\prime}\right)\right)\right. \\
F u(t) & =\left(-\int_{0}^{t} q(x) h_{n_{0}}^{*}\left(x, u(x), u^{\Delta}(x)\right) \nabla x, \sum_{i=1}^{m_{2}} \psi_{i}^{*}\left(u^{\Delta}\left(\xi_{i}\right)\right)+\rho_{n_{0}}\right) .
\end{aligned}
$$

It follows from the Arzela-Ascoli theorem on time scales [30] that $F$ is continuous and compact. Also if

$$
L_{p} v=(u, \gamma), \quad \text { for } u \in C_{0}[0, T]_{\mathbb{T}}, \gamma=\sum_{i=1}^{m_{1}} \phi_{j}^{*}\left(\rho_{n_{0}}+\int_{0}^{\xi_{j}^{\prime}} \varphi_{q}\left(u(x)-\sum_{j=1}^{m_{1}} \phi_{j}^{*}\left(u\left(\xi_{j}^{\prime}\right)\right) \Delta x\right)\right.
$$

then we have $v(t)=\rho_{n_{0}}+\int_{0}^{t} \varphi_{q}\left(u(x)-\sum_{j=1}^{m_{1}} \phi_{j}^{*}\left(u\left(\xi_{j}^{\prime}\right)\right)\right) \Delta x$. Hence $L_{p}^{-1}$ exists and is continuous. It is clear that solving the boundary value problem (2.8) and (2.9) is equivalent to finding a fixed point of $u=L_{p}^{-1} F u \equiv N u$, where $N=L_{p}^{-1} F: C_{\rho_{n_{0}}}^{\Delta}[0, T]_{\mathbb{T}^{\kappa}} \rightarrow C_{\rho_{n_{0}}}^{\Delta}[0, T]_{\mathbb{T}^{\kappa}}$ is compact. It follows from Schauder's fixed point theorem that the boundary value problem (2.8) and (2.9) has a solution $u_{n_{0}}(t) \in C^{\Delta}[0, T]_{\mathbb{T}^{\kappa}}$ with $\varphi_{p}\left(u_{n_{0}}^{\Delta}(t)\right) \in C^{\nabla}(0, T)_{\mathbb{T}}$.

In the following, we will show that

$$
\alpha_{n_{0}}(t) \leq u_{n_{0}}(t), \quad \text { for } t \in[0, T]_{\mathbb{T}} .
$$

Assume that (2.17) is not true, then the function $u_{n_{0}}(t)-\alpha_{n_{0}}(t)$ has a negative minimum for some $\tau \in(0, T]_{\mathbb{T}}$. We consider two cases, that is, $\tau \in(0, T)_{\mathbb{T}}$ and $\tau=T$.

Case 1. Assume that $\tau \in(0, T)_{\mathbb{T}}$, then we claim

$$
\left(\varphi_{p}\left(u_{n_{0}}^{\Delta}\right)\right)^{\nabla}(\tau) \geq\left(\varphi_{p}\left(\alpha_{n_{0}}^{\Delta}\right)\right)^{\nabla}(\tau)
$$

Since $u_{n_{0}}(t)-\alpha_{n_{0}}(t)$ has a negative minimum for some $\tau \in(0, T)_{\mathbb{T}}$, we have $u_{n_{0}}^{\Delta}(\tau)-\alpha_{n_{0}}^{\Delta}(\tau) \geq 0$, and there exists a $\delta$ with $\tau-\delta \in[0, \tau)_{\mathbb{T}}$ such that $u_{n_{0}}^{\Delta}(t)-\alpha_{n_{0}}^{\Delta}(t) \leq 0$ for $t \in[\tau-\delta, \tau)_{\mathbb{T}}$. Thus

$$
\varphi_{p}\left(u_{n_{0}}^{\Delta}(t)\right)-\varphi_{p}\left(\alpha_{n_{0}}^{\Delta}(t)\right) \leq \varphi_{p}\left(u_{n_{0}}^{\Delta}(\tau)\right)-\varphi_{p}\left(\alpha_{n_{0}}^{\Delta}(\tau)\right), \quad \text { for } t \in[\tau-\delta, \tau)_{\mathbb{T}}
$$

which leads to

$$
\frac{\varphi_{p}\left(u_{n_{0}}^{\Delta}(t)\right)-\varphi_{p}\left(u_{n_{0}}^{\Delta}(\tau)\right)}{t-\tau} \geq \frac{\varphi_{p}\left(\alpha_{n_{0}}^{\Delta}(t)\right)-\varphi_{p}\left(\alpha_{n_{0}}^{\Delta}(\tau)\right)}{t-\tau}, \quad \text { for } t \in[\tau-\delta, \tau)_{\mathbb{T}} .
$$


If $\tau$ is left dense, then

$$
\begin{aligned}
\left(\varphi_{p}\left(u_{n_{0}}^{\Delta}\right)\right)^{\nabla}(\tau) & =\lim _{t \in[\tau-\delta, \tau) \rightarrow \tau} \frac{\varphi_{p}\left(u_{n_{0}}^{\Delta}(t)\right)-\varphi_{p}\left(u_{n_{0}}^{\Delta}(\tau)\right)}{t-\tau} \\
& \geq \lim _{t \in[\tau-\delta, \tau) \rightarrow \tau} \frac{\varphi_{p}\left(\alpha_{n_{0}}^{\Delta}(t)\right)-\varphi_{p}\left(\alpha_{n_{0}}^{\Delta}(\tau)\right)}{t-\tau}=\left(\varphi_{p}\left(\alpha_{n_{0}}^{\Delta}\right)\right)^{\nabla}(\tau) .
\end{aligned}
$$

If $\tau$ is left scattered, then, by means of (2.20) we obtain

$$
\begin{aligned}
\left(\varphi_{p}\left(u_{n_{0}}^{\Delta}\right)\right)^{\nabla}(\tau) & =\frac{\varphi_{p}\left(u_{n_{0}}^{\Delta}(\tau)\right)-\varphi_{p}\left(u_{n_{0}}^{\Delta}(\rho(\tau))\right)}{\tau-\rho(\tau)} \\
& \geq \frac{\varphi_{p}\left(\alpha_{n_{0}}^{\Delta}(\tau)\right)-\varphi_{p}\left(\alpha_{n_{0}}^{\Delta}(\rho(\tau))\right)}{\tau-\rho(\tau)}=\left(\varphi_{p}\left(\alpha_{n_{0}}^{\Delta}\right)\right)^{\nabla}(\tau)
\end{aligned}
$$

Hence, (2.18) is true.

However, by (2.4), (2.10), and $u_{n_{0}}(\tau)<\alpha_{n_{0}}(\tau)$, we obtain

$$
\begin{aligned}
\left(\varphi_{p}\left(u_{n_{0}}^{\Delta}(\tau)\right)\right)^{\nabla}-\left(\varphi_{p}\left(\alpha_{n_{0}}^{\Delta}(\tau)\right)\right)^{\nabla} \\
\quad=-\left[q(\tau) h_{n_{0}}\left(\tau, \alpha_{n_{0}}(\tau), \alpha_{n_{0}}^{\Delta}(\tau)\right)+q(\tau) r\left(\alpha_{n_{0}}(\tau)-u_{n_{0}}(\tau)\right)+\left(\varphi_{p}\left(\alpha_{n_{0}}^{\Delta}(\tau)\right)\right)^{\nabla}\right] \\
\quad= \begin{cases}-\left[q(\tau) h_{n_{0}}\left(\tau, \alpha(\tau), \alpha^{\Delta}(\tau)\right)+q(\tau) r\left(\alpha(\tau)-u_{n_{0}}(\tau)\right)+\left(\varphi_{p}\left(\alpha^{\Delta}(\tau)\right)\right)^{\nabla}\right], & \tau \in\left[t_{n_{0}}, T\right)_{\mathbb{T}}, \\
-\left[q(\tau) h_{n_{0}}\left(\tau, \rho_{n_{0}}, 0\right)+q(\tau) r\left(\rho_{n_{0}}-u_{n_{0}}(\tau)\right)\right], & \tau \in\left(0, t_{n_{0}}\right)_{\mathbb{T}} .\end{cases}
\end{aligned}
$$

Assume that $\tau \in\left[1 / 2^{n_{0}+1}, T-\left(1 / 2^{n_{0}+1}\right)\right]_{\mathbb{T}}$, then $h_{n_{0}}(\tau, x, y)=f(\tau, x, y)$ for $(x, y) \in$ $(0, \infty) \times(-\infty,+\infty)$. It follows from (A1) and (A2) that

$$
\begin{aligned}
& \left(\varphi_{p}\left(u_{n_{0}}^{\Delta}(\tau)\right)\right)^{\nabla}-\left(\varphi_{p}\left(\alpha_{n_{0}}^{\Delta}(\tau)\right)\right)^{\nabla} \\
& \quad= \begin{cases}-\left[q(\tau) f\left(\tau, \alpha(\tau), \alpha^{\Delta}(\tau)\right)+q(\tau) r\left(\alpha(\tau)-u_{n_{0}}(\tau)\right)+\left(\varphi_{p}\left(\alpha^{\Delta}(\tau)\right)\right)^{\nabla}\right], & \tau \in\left[t_{n_{0}}, T\right)_{\mathbb{T}} \\
-\left[q(\tau) f\left(\tau, \rho_{n_{0}}, 0\right)+q(\tau) r\left(\rho_{n_{0}}-u_{n_{0}}(\tau)\right)\right], & \tau \in\left(0, t_{n_{0}}\right)_{\mathbb{T}}\end{cases} \\
& \quad<0 .
\end{aligned}
$$

This is a contraction. 
Assume that $\tau \in\left(0,\left(1 / 2^{n_{0}+1}\right)\right)_{\mathbb{T}} \cup\left(T-\left(1 / 2^{n_{0}+1}\right), T\right)_{\mathbb{T}}$, then

$$
\begin{aligned}
h_{n_{0}}(\tau, x, y) & =f_{n_{0}}(\tau, x, y)=\max \left\{f(\tau, x, y), f\left(\omega_{n_{0}}(\tau), x, y\right)\right\} \\
& =\max \left\{f\left(\frac{1}{2^{n_{0}+1}}, x, y\right), f\left(T-\frac{1}{2^{n_{0}+1}}, x, y\right), f(\tau, x, y)\right\},
\end{aligned}
$$

in view of (A1), (A2) and $q(\tau)>0$, we have

$$
\begin{aligned}
& \left(\varphi_{p}\left(u_{n_{0}}^{\Delta}(\tau)\right)\right)^{\nabla}-\left(\varphi_{p}\left(\alpha_{n_{0}}^{\Delta}(\tau)\right)\right)^{\nabla} \\
& \quad \leq \begin{cases}-\left[q(\tau) f\left(\tau, \alpha(\tau), \alpha^{\Delta}(\tau)\right)+\left(\varphi_{p}\left(\alpha^{\Delta}(\tau)\right)\right)^{\nabla}\right] & \tau \in\left[t_{n_{0}}, T-\frac{1}{2^{n_{0}+1}}\right)_{\mathbb{T}^{\prime}} \\
-q(\tau) r\left(\alpha(\tau)-u_{n_{0}}(\tau)\right), & \tau \in\left(0, t_{n_{0}}\right)_{\mathbb{T}}, \\
-\left[q(\tau) f\left(\frac{1}{2^{n_{0}+1}}, \rho_{n_{0}}, 0\right)+q(\tau) r\left(\rho_{n_{0}}-u_{n_{0}}(\tau)\right)\right], & \\
-\left[q(\tau) f\left(T-\frac{1}{2^{n_{0}+1}}, \rho_{n_{0}}, 0\right)+q(\tau) r\left(\rho_{n_{0}}-u_{n_{0}}(\tau)\right)\right], & \tau \in\left[T-\frac{1}{2^{n_{0}+1}}, T\right)_{\mathbb{T}^{\prime}},\end{cases}
\end{aligned}
$$

$<0$.

This is a contraction.

Case 2. Assume that $\tau=T$. That is, $\alpha_{n_{0}}(T)-u_{n_{0}}(T)>0$, this implies $\phi_{m_{1}}\left(\alpha_{n_{0}}(T)\right)-\phi_{m_{1}}\left(u_{n_{0}}(T)\right)>$ 0 .

From (2.4), (2.9), (2.11), (2.12), and $\sum_{j=1}^{m_{1}} \phi_{j}\left(\alpha\left(\xi_{j}^{\prime}\right)\right) \leq \sum_{i=1}^{m_{2}} \psi_{j}\left(\alpha^{\Delta}\left(\xi_{i}\right)\right)$, we have the following three subcases.

(a) If $u_{n_{0}}\left(\xi_{j}^{\prime}\right) \leq \alpha\left(\xi_{j}^{\prime}\right)$ for $j=1,2, \ldots, m_{1}-1$ and $u_{n_{0}}^{\Delta}\left(\xi_{i}\right) \leq \alpha^{\Delta}\left(\xi_{i}\right)$ for $i=1,2, \ldots, m_{2}$, then

$$
\begin{aligned}
0 & <\phi_{m_{1}}\left(\alpha_{n_{0}}(T)\right)-\phi_{m_{1}}\left(u_{n_{0}}(T)\right)=\phi_{m_{1}}(\alpha(T))-\phi_{m_{1}}\left(u_{n_{0}}(T)\right) \\
& \leq-\sum_{j=1}^{m_{1}-1} \phi_{j}\left(\alpha\left(\xi_{j}^{\prime}\right)\right)+\sum_{j=1}^{m_{2}} \psi_{i}\left(\alpha^{\Delta}\left(\xi_{i}\right)\right)+\sum_{j=1}^{m_{1}-1} \phi_{j}^{*}\left(u_{n_{0}}\left(\xi_{j}^{\prime}\right)\right)-\sum_{i=1}^{m_{2}} \psi_{i}^{*}\left(u_{n_{0}}^{\Delta}\left(\xi_{i}\right)\right)-\rho_{n_{0}} \\
& <-\sum_{j=1}^{m_{1}-1} \phi_{j}\left(\alpha\left(\xi_{j}^{\prime}\right)\right)+\sum_{i=1}^{m_{2}} \psi_{i}\left(\alpha^{\Delta}\left(\xi_{i}\right)\right)+\sum_{j=1}^{m_{1}-1} \phi_{j}\left(\alpha\left(\xi_{j}^{\prime}\right)\right)-\sum_{i=1}^{m_{2}} \psi_{i}\left(\alpha^{\Delta}\left(\xi_{i}\right)\right)=0
\end{aligned}
$$

this is a contradiction.

(b) If $\alpha\left(\xi_{j}^{\prime}\right) \leq u_{n_{0}}\left(\xi_{j}^{\prime}\right)$ for $j=1,2, \ldots, m_{1}-1$ and $\alpha^{\Delta}\left(\xi_{i}\right) \leq u_{n_{0}}^{\Delta}\left(\xi_{i}\right)$ for $i=1,2, \ldots, m_{2}$, then we discuss the four subcases.

Assume that $u_{n_{0}}\left(\xi_{j}^{\prime}\right) \leq \beta\left(\xi_{j}^{\prime}\right)$ for $j=1,2, \ldots, m_{1}-1$, then

$$
\sum_{j=1}^{m_{1}-1} \phi_{j}^{*}\left(u_{n_{0}}\left(\xi_{j}^{\prime}\right)\right)=\sum_{j=1}^{m_{1}-1} \phi_{j}\left(u_{n_{0}}\left(\xi_{j}^{\prime}\right)\right) .
$$


Advances in Difference Equations

Assume that $\beta\left(\xi_{j}^{\prime}\right)<u_{n_{0}}\left(\xi_{j}^{\prime}\right)$ for $i=1,2, \ldots, m_{1}-1$, then

$$
\sum_{j=1}^{m_{1}-1} \phi_{j}^{*}\left(u_{n_{0}}\left(\xi_{j}^{\prime}\right)\right)=\sum_{j=1}^{m_{1}-1} \phi_{j}\left(\beta\left(\xi_{j}^{\prime}\right)\right)
$$

Assume that $u_{n_{0}}^{\Delta}\left(\xi_{i}\right) \leq \beta^{\Delta}\left(\xi_{i}\right)$ for $i=1,2, \ldots, m_{2}$, then

$$
\sum_{i=1}^{m_{2}} \psi_{i}^{*}\left(u_{n_{0}}^{\Delta}\left(\xi_{i}\right)\right)=\sum_{i=1}^{m_{2}} \psi_{i}\left(u_{n_{0}}^{\Delta}\left(\xi_{i}\right)\right)
$$

Assume that $\beta^{\Delta}\left(\xi_{i}\right)<u_{n_{0}}^{\Delta}\left(\xi_{i}\right)$ for $i=1,2, \ldots, m_{2}$, then

$$
\sum_{i=1}^{m_{2}} \psi_{i}^{*}\left(u_{n_{0}}^{\Delta}\left(\xi_{i}\right)\right)=\sum_{i=1}^{m_{2}} \psi_{i}\left(\beta^{\Delta}\left(\xi_{i}\right)\right)
$$

Now, if there exist sequences $\left\{j_{l_{1}}\right\}=\left\{1,2, \ldots, l_{1}\right\}$ and $\left\{j_{k_{1}}\right\}=\left\{1,2, \ldots, k_{1}\right\}$ such that $\beta\left(\xi_{j_{1}}^{\prime}\right)<u_{n_{0}}\left(\xi_{j_{l}}^{\prime}\right)$ and $u_{n_{0}}\left(\xi_{j_{k_{1}}}^{\prime}\right) \leq \beta\left(\xi_{j_{k_{1}}}^{\prime}\right)$, here $l_{1}+k_{1}=m_{1}-1$, then

$$
\sum_{j=1}^{m_{1}-1} \phi_{j}^{*}\left(u_{n_{0}}\left(\xi_{j}^{\prime}\right)\right)=\sum_{j_{l_{1}}=1}^{l_{1}} \phi_{j_{l_{1}}}\left(\beta\left(\xi_{j_{1}}^{\prime}\right)\right)+\sum_{j_{k_{1}}=1}^{k_{1}} \phi_{j_{k_{1}}}\left(u_{n_{0}}\left(\xi_{j_{k_{1}}}^{\prime}\right)\right) .
$$

If there exist sequences $\left\{i_{l_{2}}\right\}=\left\{1,2, \ldots, l_{2}\right\}$ and $\left\{i_{k_{2}}\right\}=\left\{1,2, \ldots, k_{2}\right\}$ such that $\beta^{\Delta}\left(\xi_{i_{2}}\right)<$ $u_{n_{0}}^{\Delta}\left(\xi_{i_{2}}\right)$ and $u_{n_{0}}^{\Delta}\left(\xi_{i_{k_{2}}}\right) \leq \beta^{\Delta}\left(\xi_{i_{k_{2}}}\right)$, here $l_{2}+k_{2}=m_{2}$, then

$$
\sum_{i=1}^{m_{2}} \psi_{i}^{*}\left(u_{n_{0}}^{\Delta}\left(\xi_{i}\right)\right)=\sum_{i_{l_{2}}=1}^{l_{2}} \psi_{i_{l_{2}}}\left(\beta^{\Delta}\left(\xi_{i_{l_{2}}}\right)\right)+\sum_{i_{k_{2}}=1}^{k_{2}} \psi_{i_{k_{2}}}\left(u_{n_{0}}^{\Delta}\left(\xi_{i_{k_{2}}}\right)\right) .
$$

Hence, by (2.32) and (2.33) together with the monotonicity of $\phi_{j}$ and $\psi_{i}$, we have

$$
\begin{aligned}
0 & <\phi_{m_{1}}\left(\alpha_{n_{0}}(T)\right)-\phi_{m_{1}}\left(u_{n_{0}}(T)\right)=\phi_{m_{1}}(\alpha(T))-\phi_{m_{1}}\left(u_{n_{0}}(T)\right) \\
& \leq-\sum_{j=1}^{m_{1}-1} \phi_{j}\left(\alpha\left(\xi_{j}^{\prime}\right)\right)+\sum_{j=1}^{m_{2}} \psi_{i}\left(\alpha^{\Delta}\left(\xi_{i}\right)\right)+\sum_{j=1}^{m_{1}-1} \phi_{j}^{*}\left(u_{n_{0}}\left(\xi_{j}^{\prime}\right)\right)-\sum_{i=1}^{m_{2}} \psi_{i}^{*}\left(u_{n_{0}}^{\Delta}\left(\xi_{i}\right)\right)-\rho_{n_{0}} \\
& <0
\end{aligned}
$$

which implies a contradiction.

(c) For $j=1,2, \ldots, m_{1}-1$, if there exist sequences $\left\{j_{l_{3}}\right\}=\left\{1,2, \ldots, l_{3}\right\}$ and $\left\{j_{k_{3}}\right\}=$ $\left\{1,2, \ldots, k_{3}\right\}$ such that $\alpha\left(\xi_{j_{l_{3}}}^{\prime}\right) \leq u_{n_{0}}\left(\xi_{j_{l_{3}}}^{\prime}\right)$ and $u_{n_{0}}\left(\xi_{j_{k_{3}}}^{\prime}\right) \leq \alpha\left(\xi_{j_{k_{3}}}^{\prime}\right)$, here $l_{3}+k_{3}=m_{1}-1$. For $i=1,2, \ldots, m_{2}$, if there exist sequences $\left\{i_{l}\right\}=\{1,2, \ldots, l\}$ and $\left\{i_{k}\right\}=\{1,2, \ldots, k\}$ such that 
$\alpha^{\Delta}\left(\xi_{i_{l}}\right) \leq u_{n_{0}}^{\Delta}\left(\xi_{i_{l}}\right)$ and $u_{n_{0}}^{\Delta}\left(\xi_{i_{k}}\right) \leq \alpha^{\Delta}\left(\xi_{i_{k}}\right)$, here $l+k=m_{2}$. Essentially, with the same reasoning as before, we have

$$
0<\phi_{m_{1}}\left(\alpha_{n_{0}}(T)\right)-\phi_{m_{1}}\left(u_{n_{0}}(T)\right)=\phi_{m_{1}}(\alpha(T))-\phi_{m_{1}}\left(u_{n_{0}}(T)\right)<0,
$$

which implies a contradiction.

Thus, Cases 1 and 2 imply that (2.17) is established. In particular, since $\alpha(t) \leq \alpha_{n_{0}}(t)$ for $t \in[0, T]_{\mathbb{T}}$, we obtain $\alpha(t) \leq \alpha_{n_{0}}(t) \leq u_{n_{0}}(\mathrm{t})$ for $t \in[0, T]_{\mathbb{T}}$.

Essentially, with the same reasoning as the proof of inequality (2.17), we obtain $u_{n_{0}}(t) \leq$ $\beta(t)$ for $t \in[0, T]_{\mathbb{T}}$.

Hence

$$
\alpha(t) \leq \alpha_{n_{0}}(t) \leq u_{n_{0}}(t) \leq \beta(t) \text { for } t \in[0, T]_{\mathbb{T}} .
$$

Now, we discuss the boundary value problem

$$
\begin{gathered}
\left(\varphi_{p}\left(u^{\Delta}(t)\right)\right)^{\nabla}+q(t) h_{n_{0}+1}^{*}\left(t, u(t), u^{\Delta}(t)\right)=0, \quad t \in(0, T)_{\mathbb{T}}, \\
u(0)=\rho_{n_{0}+1}, \quad \sum_{j=1}^{m_{1}} \phi_{j}^{*}\left(u\left(\xi_{j}^{\prime}\right)\right)-\sum_{i=1}^{m_{2}} \psi_{i}^{*}\left(u^{\Delta}\left(\xi_{i}\right)\right)=\rho_{n_{0}+1},
\end{gathered}
$$

where

$$
\begin{aligned}
& h_{n_{0}+1}^{*}\left(t, u(t), u^{\Delta}(t)\right) \\
& = \begin{cases}h_{n_{0}+1}\left(t, \alpha_{n_{0}+1}(t), \alpha_{n_{0}+1}^{\Delta}(t)\right)+r\left(\alpha_{n_{0}+1}(t)-u(t)\right), & u(t) \leq \alpha_{n_{0}+1}(t), \\
h_{n_{0}+1}\left(t, u(t), u^{\Delta}(t)\right), & \alpha_{n_{0}+1}(t) \leq u(t) \leq u_{n_{0}}(t), \\
h_{n_{0}+1}\left(t, u_{n_{0}}(t), u_{n_{0}}^{\Delta}(t)\right)+r\left(u_{n_{0}}(t)-u(t)\right), & u(t) \geq u_{n_{0}}(t),\end{cases} \\
& \phi_{j}^{*}\left(z_{j}^{\prime}\right)= \begin{cases}\phi_{j}\left(\alpha\left(\xi_{j}^{\prime}\right)\right), & z_{j}^{\prime} \leq \alpha_{n_{0}+1}\left(\xi_{j}^{\prime}\right)=\alpha\left(\xi_{j}^{\prime}\right), \\
\phi_{j}\left(z_{j}^{\prime}\right), & \alpha_{n_{0}+1}\left(\xi_{j}^{\prime}\right) \leq z_{j}^{\prime} \leq u_{n_{0}}\left(\xi_{j}^{\prime}\right), \quad j=1, \ldots, m_{1}-1, \\
\phi_{j}\left(u_{n_{0}}\left(\xi_{j}^{\prime}\right)\right), & z_{j}^{\prime} \geq u_{n_{0}}\left(\xi_{j}^{\prime}\right),\end{cases} \\
& \psi_{i}^{*}\left(z_{i}\right)= \begin{cases}\psi_{i}\left(\alpha^{\Delta}\left(\xi_{i}\right)\right), & z_{i} \leq \alpha_{n_{0}+1}^{\Delta}\left(\xi_{i}\right)=\alpha^{\Delta}\left(\xi_{i}\right), \\
\psi_{i}\left(z_{i}\right), & \alpha_{n_{0}+1}^{\Delta}\left(\xi_{i}\right) \leq z_{i} \leq u_{n_{0}}^{\Delta}\left(\xi_{i}\right), \quad i=1, \ldots, m_{2} . \\
\psi_{i}\left(u_{n_{0}}^{\Delta}\left(\xi_{i}\right)\right), & z_{i} \geq u_{n_{0}}^{\Delta}\left(\xi_{i}\right),\end{cases}
\end{aligned}
$$

It follows from Schauder's fixed point theorem that the boundary value problem (2.37) has a solution $u_{n_{0}+1}(t) \in C^{\Delta}[0, T]_{\mathbb{T}^{\kappa}}$ with $\varphi_{p}\left(u_{n_{0}+1}^{\Delta}(t)\right) \in C^{\nabla}(0, T)_{\mathbb{T}}$.

Essentially, with the same reasoning as the proof of inequality (2.36), we have

$$
\alpha(t) \leq \alpha_{n_{0}+1}(t) \leq u_{n_{0}+1}(t) \leq u_{n_{0}}(t) \quad \text { for } t \in[0, T]_{\mathbb{T}} .
$$


Assume that we have $u_{k}(t)$ for some $k \in\left\{n_{0}+1, n_{0}+2, \ldots\right\}$ satisfying $\alpha_{k}(t) \leq u_{k}(t) \leq$ $u_{k-1}(t)$ for $t \in[0, T]_{\mathbb{T}}$, then we investigate the boundary value problem

$$
\begin{aligned}
& \left(\varphi_{p}\left(u^{\Delta}(t)\right)\right)^{\nabla}+q(t) h_{k+1}^{*}\left(t, u(t), u^{\Delta}(t)\right)=0, \quad t \in(0, T)_{\mathbb{T}}, \\
& u(0)=\rho_{k+1}, \quad \sum_{j=1}^{m_{1}} \phi_{j}\left(u\left(\xi_{j}^{\prime}\right)\right)-\sum_{i=1}^{m} \psi_{i}^{*}\left(u^{\Delta}\left(\xi_{i}\right)\right)=\rho_{k+1},
\end{aligned}
$$

where

$$
\begin{aligned}
& h_{k+1}^{*}\left(t, u(t), u^{\Delta}(t)\right) \\
& = \begin{cases}h_{k+1}\left(t, \alpha_{k+1}(t), \alpha_{k+1}^{\Delta}(t)\right)+r\left(\alpha_{k+1}(t)-u(t)\right), & u(t) \leq \alpha_{k+1}(t), \\
h_{k+1}\left(t, u(t), u^{\Delta}(t)\right), & \alpha_{k+1}(t) \leq u(t) \leq u_{k}(t), \\
h_{k+1}\left(t, u_{k}(t), u_{k}^{\Delta}(t)\right)+r\left(u_{k}(t)-u(t)\right), & u(t) \geq u_{k}(t),\end{cases} \\
& \phi_{j}^{*}\left(z_{j}^{\prime}\right)= \begin{cases}\phi_{j}\left(\alpha\left(\xi_{j}^{\prime}\right)\right), & z_{j}^{\prime} \leq \alpha_{k+1}\left(\xi_{j}^{\prime}\right)=\alpha\left(\xi_{j}^{\prime}\right), \\
\phi_{j}\left(z_{j}^{\prime}\right), & \alpha_{k+1}\left(\xi_{j}^{\prime}\right) \leq z_{j}^{\prime} \leq u_{k}\left(\xi_{j}^{\prime}\right), \quad j=1, \ldots, m_{1}-1, \\
\phi_{j}\left(u_{k}\left(\xi_{j}^{\prime}\right)\right), & z_{j}^{\prime} \geq u_{k}\left(\xi_{j}^{\prime}\right),\end{cases} \\
& \psi_{i}^{*}\left(z_{i}\right)= \begin{cases}\psi_{i}\left(\alpha^{\Delta}\left(\xi_{i}\right)\right), & z_{i} \leq \alpha_{k+1}^{\Delta}\left(\xi_{i}\right)=\alpha^{\Delta}\left(\xi_{i}\right), \\
\psi_{i}\left(z_{i}\right), & \alpha_{k+1}^{\Delta}\left(\xi_{i}\right) \leq z_{i} \leq u_{k}^{\Delta}\left(\xi_{i}\right), \quad i=1, \ldots, m_{2} . \\
\psi_{i}^{\Delta}\left(u_{k}\left(\xi_{i}\right)\right), & z_{i} \geq u_{k}^{\Delta}\left(\xi_{i}\right),\end{cases}
\end{aligned}
$$

Schauder's fixed point theorem guarantees that the boundary value problem (2.40) has a solution $u_{k+1}(t) \in C^{\Delta}[0, T]_{\mathbb{T}^{k}}$ with $\varphi_{p}\left(u_{k+1}^{\Delta}(t)\right) \in C^{\nabla}(0, T)_{\mathbb{T}}$.

By proceeding to this process as above, we have

$$
\alpha(t) \leq \alpha_{k+1}(t) \leq u_{k+1}(t) \leq u_{k}(t) \quad \text { for } t \in[0, T]_{\mathbb{T}} .
$$

Hence, for $n \in\left\{n_{0}, n_{0}+1, \ldots\right\}$, by proceeding to this process by induction, we obtain sequence $\left\{u_{n}(t)\right\}_{n \in \mathbb{N}_{1}}$ with

$$
\alpha(t) \leq \alpha_{n}(t) \leq u_{n}(t) \leq u_{n-1}(t) \leq \cdots \leq u_{n_{0}}(t) \leq \beta(t) \quad \text { for } t \in[0, T]_{\mathbb{T}} .
$$

Since $[0, T]_{\mathbb{T}}$ is compact, the convergence is monotone and bounded, there exist a subsequence $\mathbb{N}_{n_{0}}$ of integers and a function $z_{n_{0}}(t) \in C\left[1 / 2^{n_{0}+1}, T\right]_{\mathbb{T}}$ with $u_{n}(t)$ converging uniformly to $z_{n_{0}}(t)$ on $\left[1 / 2^{n_{0}+1}, T\right]_{\mathbb{T}}$ as $n \rightarrow \infty$ through $\mathbb{N}_{n_{0}}$. Similarly

$$
\left\{u_{n}(t)\right\}_{n=n_{0}+1}^{\infty} \text { is a bounded, monotoneon compact interval }\left[\frac{1}{2^{n_{0}+2}}, T\right]_{\mathbb{T}} \text {. }
$$


Thus there exist a subsequence $\mathbb{N}_{n_{0}+1}$ of $\mathbb{N}_{n_{0}}$ and a function $z_{n_{0}+1}(t) \in C\left[1 / 2^{n_{0}+2}, T\right]_{\mathbb{T}}$ with $u_{n}(t)$ converging uniformly to $z_{n_{0}+1}(t)$ on $\left[1 / 2^{n_{0}+2}, T\right]_{\mathbb{T}}$ as $n \rightarrow \infty$ through $\mathbb{N}_{n_{0}+1}$. Since $\mathbb{N}_{n_{0}+1} \subseteq$ $\mathbb{N}_{n_{0}}$, we have $z_{n_{0}+1}(t)=z_{n_{0}}(t)$ on $\left[1 / 2^{n_{0}+1}, T\right]_{\mathbb{T}}$. Proceed inductively to obtain subsequence of integers $\mathbb{N}_{n_{0}} \supseteq \mathbb{N}_{n_{0}+1} \supseteq \cdots \supseteq \mathbb{N}_{n} \supseteq \cdots$ and functions $z_{n}(t) \in C\left[1 / 2^{n+1}, T\right]_{\mathbb{T}}$ with $u_{n}(t)$ converging uniformly to $z_{n}(t)$ on $\left[1 / 2^{n+1}, T\right]_{\mathbb{T}}$ as $n \rightarrow \infty$ through $\mathbb{N}_{n}$ and $z_{n}(t)=z_{n-1}(t)$ on $\left[1 / 2^{n}, T\right]_{\mathbb{T}}$.

Now, we define a function $u:[0, T]_{\mathbb{T}} \rightarrow[0, \infty)$ with $u(t)=z_{n}(t)$ on $\left[1 / 2^{n+1}, T\right]_{\mathbb{T}}$ and $u(0)=0$. Notice, $u(t)$ is well defined and $\alpha(t) \leq u(t) \leq u_{n_{0}}(t) \leq \beta(t)$ for $t \in(0, T)_{\mathbb{T}}$. Nextly, fix $t \in(0, T)_{\mathbb{T}}$ and let $l \in\left\{n_{0}, n_{0}+1, \ldots\right\}$ be such that $t \in\left(1 / 2^{l+1}, T\right)_{\mathbb{T}}$, assume that $\mathbb{N}_{l}^{*}=\left\{n \in \mathbb{N}_{l}: n \geq l\right\}$, we have

$$
\begin{gathered}
\phi_{j}^{*}\left(u_{n}\left(\xi_{j}^{\prime}\right)\right)=\phi_{j}\left(u_{n}\left(\xi_{j}^{\prime}\right)\right) \quad \text { for } j=1,2, \ldots, m_{1}, \\
\psi_{i}^{*}\left(u_{n}\left(\xi_{i}\right)\right)=\psi_{i}\left(u_{n}\left(\xi_{i}\right)\right) \quad \text { for } i=1,2, \ldots, m_{2}, \\
h_{n}^{*}\left(t, u_{n}(t), u_{n}^{\Delta}(t)\right)=h_{n}\left(t, u_{n}(t), u_{n}^{\Delta}(t)\right)=f\left(t, u_{n}(t), u_{n}^{\Delta}(t)\right) \quad \text { for } n \in \mathbb{N}_{l}^{*} .
\end{gathered}
$$

Hence, for $n \in \mathbb{N}_{l}^{*}$, we have $u_{n}$ as the positive solution of the following boundary value problem:

$$
\begin{gathered}
\left(\varphi_{p}\left(u_{n}^{\Delta}(t)\right)\right)^{\nabla}+q(t) f\left(t, u_{n}(t), u_{n}^{\Delta}(t)\right)=0, \quad t \in\left(\frac{1}{2^{n+1}}, T\right)_{\mathbb{T}}, \\
u_{n}(0)=\rho_{n}, \quad \sum_{j=1}^{m_{1}} \phi_{j}\left(u_{n}\left(\xi_{j}^{\prime}\right)\right)-\sum_{i=1}^{m_{2}} \psi_{i}\left(u_{n}^{\Delta}\left(\xi_{i}\right)\right)=\rho_{n} .
\end{gathered}
$$

Let $n \rightarrow \infty$ through $\mathbb{N}_{l}^{*}$, we obtain that $u(t)$ satisfies

$$
\begin{gathered}
\left(\varphi_{p}\left(u^{\Delta}(t)\right)\right)^{\nabla}+q(t) f\left(t, u(t), u^{\Delta}(t)\right)=0, \quad t \in(0, T)_{\mathbb{T}}, \\
u(0)=0, \quad \sum_{j=1}^{m_{1}} \phi_{j}\left(u\left(\xi_{j}^{\prime}\right)\right)-\sum_{i=1}^{m_{2}} \psi_{i}\left(u^{\Delta}\left(\xi_{i}\right)\right)=0 .
\end{gathered}
$$

It remains to show that $u(t)$ is continuous at 0 .

First, it follows from $\lim _{n \rightarrow \infty} u_{n}(0)=0$ that there exists $n_{1} \in\left\{n_{0}, n_{0}+1, \ldots\right\}$ with $u_{n_{1}}(0)<$ $\varepsilon / 2$. Since $u_{n_{1}}(t) \in C[0, T]_{\mathbb{T}}$, there exists $\delta_{n_{1}} \in(0, T)_{\mathbb{T}}$ with $u_{n_{1}}(t)<\varepsilon / 2$ for $t \in\left[0, \delta_{n_{1}}\right)_{\mathbb{T}}$. By the monotonicity of $\left\{u_{n}(t)\right\}_{n \in \mathbb{N}_{0}}$ for each $t \in[0, T]_{\mathbb{T}}$, we have $\alpha(t) \leq u_{n}(t) \leq u_{n_{1}}(t)<\varepsilon / 2$ for $t \in\left[0, \delta_{n_{1}}\right)_{\mathbb{T}}$ and $n \geq n_{1}$, which means $\alpha(t) \leq u(t)<\varepsilon / 2$ for $t \in\left[0, \delta_{n_{1}}\right)_{\mathbb{T}}$. So $u(t)$ is continuous at 0 .

If we replace $\left[1 / 2^{n+1}, T-\left(1 / 2^{n+1}\right)\right]_{\mathbb{T}}$ with $t \in\left[0, T-\left(1 / 2^{n+1}\right)\right]_{\mathbb{T}}$, then the singularity occurs at $u=0$ and $t=T$.

If we replace $\left[1 / 2^{n+1}, T-\left(1 / 2^{n+1}\right)\right]_{\mathbb{T}}$ with $t \in\left[1 / 2^{n+1}, T\right]_{\mathbb{T}^{x}}$, then the singularity occurs at $u=0$ and $t=0$. 

$u=0$.

If we replace $\left[1 / 2^{n+1}, T-\left(1 / 2^{n+1}\right)\right]_{\mathbb{T}}$ with $t \in[0, T]_{\mathbb{T}^{x}}$, then the singularity occurs at remark.

So we easily obtain the analogue of Theorem 2.1 in this section; see the following

Remark 2.2. (A3) is appropriately adjusted, then we can replace $t \in\left[1 / 2^{n+1}, T-\left(1 / 2^{n+1}\right)\right]_{\mathbb{T}}$ in (A1) by

$$
\begin{aligned}
& t \in\left[0, T-\frac{1}{2^{n+1}}\right]_{\mathbb{T}^{\prime}} \\
& t \in\left[\frac{1}{2^{n+1}}, T\right]_{\mathbb{T}^{\kappa}},
\end{aligned}
$$

or

$$
t \in[0, T]_{\mathbb{T}^{\kappa}}
$$

For example, if (2.49) occurs, then (A3) is replaced by the following.

(A3') There exists a function $\beta(t) \in C[0, T]_{\mathbb{T}} \cap C^{\Delta}(0, T]_{\mathbb{T}^{\kappa}}, \varphi_{p}\left(\beta^{\Delta}(t)\right) \in C^{\nabla}(0, T)_{\mathbb{T}}$ satisfies $\beta(t) \geq \alpha(t), \beta(t) \geq \rho_{n_{0}}$ for $t \in[0, T]_{\mathbb{T}}$ and $\sum_{j=1}^{m_{1}} \phi_{j}\left(\beta\left(\xi_{j}^{\prime}\right)\right)-\sum_{i=1}^{m_{2}} \psi_{i}\left(\beta^{\Delta}\left(\xi_{i}\right)\right)>0$, with $-\left(\varphi_{p}\left(\beta^{\Delta}(t)\right)\right)^{\nabla} \geq q(t) f\left(t, \beta(t), \beta^{\Delta}(t)\right)$ for $t \in(0, T)_{\mathbb{T}}$, and $-\left(\varphi_{p}\left(\beta^{\Delta}(t)\right)\right)^{\nabla} \geq$ $q(t) f\left(1 / 2^{n_{0}+1}, \beta(t), \beta^{\Delta}(t)\right)$ for $t \in\left(0,1 / 2^{n_{0}+1}\right)_{\mathbb{T}}$.

Assume that (H1)-(H3), (A1), and (A2) hold, and in addition suppose that the following conditions are satisfied.

(A4) $-\left(\varphi_{p}\left(\alpha^{\Delta}(t)\right)\right)^{\nabla}<q(t) f\left(t, u(t), u^{\Delta}(t)\right)$ for $\left(t, u(t), u^{\Delta}(t)\right) \in(0, T)_{\mathbb{T}} \times(0, \alpha(t)] \times$ $(-\infty,+\infty)$, here $u(t) \in C[0, T]_{\mathbb{T}} \cap C^{\Delta}(0, T]_{\mathbb{T}^{\kappa}}$.

(A5) There exists a function $\beta(t) \in C[0, T]_{\mathbb{T}} \cap C^{\Delta}(0, T]_{\mathbb{T}^{x}}, \varphi_{p}\left(\beta^{\Delta}(t)\right) \in C^{\nabla}(0, T)_{\mathbb{T}}$ such that $\beta(t) \geq \rho_{n_{0}}$ for $t \in[0, T]_{\mathbb{T}}, \sum_{j=1}^{m_{1}} \psi_{i}\left(\beta\left(\xi_{j}^{\prime}\right)\right)-\sum_{i=1}^{m_{2}} \psi_{i}\left(\beta^{\Delta}\left(\xi_{i}\right)\right)>0,-\left(\varphi_{p}\left(\beta^{\Delta}(t)\right)\right)^{\nabla} \geq$ $q(t) f\left(t, \beta(t), \beta^{\Delta}(t)\right)$ for $t \in(0, T)_{\mathbb{T}}$ and

$$
\begin{aligned}
& -\left(\varphi_{p}\left(\beta^{\Delta}(t)\right)\right)^{\nabla} \geq q(t) f\left(\frac{1}{2^{n_{0}+1}}, \beta(t), \beta^{\Delta}(t)\right) \text { for } t \in\left(0, \frac{1}{2^{n_{0}+1}}\right)_{\mathbb{T}^{\prime}} \\
& -\left(\varphi_{p}\left(\beta^{\Delta}(t)\right)\right)^{\nabla} \geq q(t) f\left(T-\frac{1}{2^{n_{0}+1}}, \beta(t), \beta^{\Delta}(t)\right) \text { for } t \in\left(T-\frac{1}{2^{n_{0}+1}}, T\right)_{\mathbb{T}} \text {. }
\end{aligned}
$$

(A6) $\beta(T) \geq \alpha(T)$.

Then the result in Theorem 2.1 is also true. This follows immediately from Theorem 2.1 if we show that (A3) holds. That is to say, if we show $\beta(t) \geq \alpha(t)$ for $t \in[0, T]_{\mathbb{T}}$, then the result holds. Assume that it is not true, in view of (A6), we obtain that $\beta(t)-\alpha(t)$ has a negative minimum for some $\tau_{6} \in(0, T)_{\mathbb{T}}$, so $(\beta-\alpha)^{\Delta}\left(\tau_{6}\right) \geq 0$ and essentially the same reasoning as the proof of inequality (2.18), we have $\left(\varphi_{p}\left(\alpha^{\Delta}\right)\right)^{\nabla}\left(\tau_{6}\right) \leq\left(\varphi_{p}\left(\beta^{\Delta}\right)\right)^{\nabla}\left(\tau_{6}\right)$. However, by (A4), (A5), and $\alpha\left(\tau_{6}\right)>\beta\left(\tau_{6}\right)>0$, we obtain $-\left(\varphi_{p}\left(\alpha^{\Delta}\right)\right)^{\nabla}\left(\tau_{6}\right)<q\left(\tau_{6}\right) f\left(\tau_{6}, \beta\left(\tau_{6}\right), \beta^{\Delta}\left(\tau_{6}\right)\right)$. Hence 
$\left(\varphi_{p}\left(\alpha^{\Delta}\right)\right)^{\nabla}\left(\tau_{6}\right)-\left(\varphi_{p}\left(\beta^{\Delta}\right)\right)^{\nabla}\left(\tau_{6}\right) \geq\left(\varphi_{p}\left(\alpha^{\Delta}\right)\right)^{\nabla}\left(\tau_{6}\right)+q\left(\tau_{6}\right) f\left(\tau_{6}, \beta\left(\tau_{6}\right), \beta^{\Delta}\left(\tau_{6}\right)\right)>0$, which implies a contradiction.

Corollary 2.3. Let $n_{0} \in\{1,2, \ldots\}$ be fixed, suppose that (H1)-(H3), (A1), (A2) and (A4)-(A6) hold, then the boundary value problem (1.2) and (1.3) has a solution $u(t) \in C[0, T]_{\mathbb{T}} \cap C^{\Delta}(0, T]_{\mathbb{T}^{\kappa}}$, $\varphi_{p}\left(u^{\Delta}(t)\right) \in C^{\nabla}(0, T)_{\mathbb{T}}$ with $u(t) \geq \alpha(t)$ for $t \in[0, T]_{\mathbb{T}}$.

\section{Construction of $\alpha(t)$ and $\beta(t)$}

In this section, we consider how to construct a lower solution $\alpha(t)$ and an upper solution $\beta(t)$ in certain conditions. In this section, we assume that

$$
\sum_{i=1}^{m_{2}} \psi_{i}\left(x_{i}\right)-\sum_{j=1}^{m_{1}-1} \phi_{j}\left(x_{j}^{\prime}\right) \geq 0 \quad \text { for } x_{i}, x_{j}^{\prime} \in \mathbb{R} .
$$

Lemma 3.1. If there exists a nonincreasing positive sequence $\left\{\varepsilon_{n}\right\}$ with $\lim _{n \rightarrow \infty} \varepsilon_{n}=0$, then there exists a function $\lambda(t) \in C^{\Delta}[0, T]_{\mathbb{T}}$ satisfying

(i) $\varphi_{p}\left(\lambda^{\Delta}(t)\right) \in C^{\nabla}[0, T]_{\mathbb{T}}, \lambda(t)>0$ for $t \in(0, T]_{\mathbb{T}}$ and $\max _{t \in[0, T]_{\mathbb{T}}}\left|\left(\varphi_{p}\left(\lambda^{\Delta}(t)\right)\right)^{\nabla}\right|>0$;

(ii) $\lambda(0)=0, \sum_{j=1}^{m_{1}} \phi_{j}\left(\lambda\left(\xi_{j}^{\prime}\right)\right)-\sum_{i=1}^{m_{2}} \psi_{i}\left(\lambda^{\Delta}\left(\xi_{i}\right)\right)<0$ and $0<\lambda(t) \leq \varepsilon_{n}$ for $t \in(0, T)_{\mathbb{T}}$.

Proof. Let $e_{n}=\left[1 / 2^{n+1}, T-\left(1 / 2^{n+1}\right)\right]_{\mathbb{T}}\left(n \geq n_{0}\right)$. Assume that $r:[0, T]_{\mathbb{T}} \rightarrow[0, \infty)$ is continuous function such that $r(0)=0, r(t)=\varepsilon_{n}^{p-1} /(2 T)^{p+1}$ for $t \in e_{n} \backslash e_{n-1}, n \geq n_{0}$, and $r(t)=\varepsilon_{n_{0}}^{p-1} /(2 T)^{p+1}$ for $t \in\left[1 / 2^{n_{0}}, T-1 / 2^{n_{0}}\right]_{\mathbb{T}}$. Let $u(t)=\int_{0}^{t} r(s) \Delta s, v(t)=\left[\int_{0}^{t} u(s) \nabla s\right]^{1 /(p-1)}$, $w(t)=\int_{0}^{t} v(s) \Delta s$. Assume that $\tau_{7} \in e_{n} \backslash e_{n-1}$ for $n \geq n_{0}, \tau_{8} \in(0, T)_{\mathbb{T}}$ with $\tau_{7}<\tau_{8}$ and $2 \tau_{8}-T \geq \tau_{7}$. It is easy to show that $u, v, w:\left[0, \tau_{7}\right]_{\mathbb{T}} \rightarrow[0, \infty)$ are continuous and increasing. Denote

$$
\begin{gathered}
a(t)=\left[c_{0}\left(\tau_{8}-t\right)+c_{1} t\right]^{1 /(p-1)} \quad \text { for } t \in\left[\tau_{7}, T\right]_{\mathbb{T}}, \\
\text { here } c_{0}=-\frac{\tau_{7}}{\tau_{8}} u\left(\tau_{7}\right)+\frac{1}{\tau_{8}}\left(v\left(\tau_{7}\right)\right)^{p-1}, \quad c_{1}=\frac{\tau_{8}-\tau_{7}}{\tau_{8}} u\left(\tau_{7}\right)+\frac{1}{\tau_{8}}\left(v\left(\tau_{7}\right)\right)^{p-1} .
\end{gathered}
$$

Hence, $a(t)>0$ for $t \in\left[\tau_{7}, T\right]_{\mathbb{T}}$ and is nondecreasing. Define

$$
\begin{gathered}
b(t)=\int_{\tau_{7}}^{t} a(s) \Delta s+w\left(\tau_{7}\right) \quad \text { for } t \in\left[\tau_{7}, \tau_{8}\right]_{\mathbb{T}}, \\
P(t)=\left\{\begin{array}{ll}
b(t), & t \in\left[\tau_{7}, \tau_{8}\right]_{\mathbb{T}}, \\
b\left(2 \tau_{8}-t\right), & t \in\left[\tau_{8}, T\right]_{\mathbb{T}},
\end{array} \quad \lambda(t)= \begin{cases}w(t), & t \in\left[0, \tau_{7}\right]_{\mathbb{T}}, \\
P(t), & t \in\left[\tau_{7}, T\right]_{\mathbb{T}} .\end{cases} \right.
\end{gathered}
$$

We can easily prove that $w\left(\tau_{7}\right)=P\left(\tau_{7}\right), w^{\Delta}\left(\tau_{7}\right)=P^{\Delta}\left(\tau_{7}\right),\left(\varphi_{p}\left(w^{\Delta}\right)\right)^{\nabla}\left(\tau_{7}\right)=\left(\varphi_{p}\left(P^{\Delta}\right)\right)^{\nabla}\left(\tau_{7}\right)$ and $w \in C^{\Delta}\left[0, \tau_{7}\right]_{\mathbb{T}}, P \in C^{\Delta}\left[\tau_{7}, T\right]_{\mathbb{T}}, \varphi_{p}\left(w^{\Delta}\right) \in C^{\nabla}\left[0, \tau_{7}\right]_{\mathbb{T}}, \varphi_{p}\left(P^{\Delta}\right) \in C^{\nabla}\left[\tau_{7}, T\right]_{\mathbb{T}}$. Thus, we have 
$\lambda \in C^{\Delta}[0, T]_{\mathbb{T}}$ and $\varphi_{p}\left(\lambda^{\Delta}\right) \in C^{\nabla}[0, T]_{\mathbb{T}}$ with $\max _{0 \leq t \leq T}\left|\left(\varphi_{p}\left(\lambda^{\Delta}\right)\right)^{\nabla}(t)\right|>0$. Now since $w(t)>0$ for $t \in\left(0, \tau_{7}\right]_{\mathbb{T}}$ and $P(t)>0$ for $t \in\left[\tau_{7}, T\right]_{\mathbb{T}}$, we have $\lambda(t)>0$ for $t \in(0, T]_{\mathbb{T}}$. On the other hand,

$$
\begin{gathered}
u\left(\tau_{7}\right)=\int_{0}^{\tau_{7}} r(s) \Delta s \leq \tau_{7} \frac{\varepsilon_{n}^{p-1}}{(2 T)^{p+1}}<\frac{\varepsilon_{n}^{p-1}}{(2 T)^{p}} \\
v\left(\tau_{7}\right)=\left[\int_{0}^{\tau_{7}} u(s) \nabla s\right]^{1 /(p-1)}<\left(\tau_{7} \frac{\varepsilon_{n}^{p-1}}{(2 T)^{p}}\right)^{1 /(p-1)}<\frac{\varepsilon_{n}}{2^{p /(p-1)} T^{\prime}} \quad w\left(\tau_{7}\right)<\tau_{7} \times \frac{\varepsilon_{n}}{2^{p /(p-1)} T}<\frac{\varepsilon_{n}}{2},
\end{gathered}
$$

by the monotonicity of $P(t)$ on $\left[\tau_{7}, \tau_{8}\right]_{\mathbb{T}},\left[\tau_{8}, T\right]_{\mathbb{T}}$, respectively, we have

$$
\begin{aligned}
\lambda\left(\tau_{8}\right) & =\max _{t \in\left[\tau_{7}, T\right]_{\mathbb{T}}} \lambda(t)=\int_{\tau_{7}}^{\tau_{8}} a(s) \Delta s+w\left(\tau_{7}\right) \\
& \leq\left(\tau_{8}-\tau_{7}\right) \max _{t \in\left[\tau_{7}, \tau_{8}\right]_{\mathbb{T}}}\left[c_{0}\left(\tau_{8}-t\right)+c_{1} t\right]^{1 /(p-1)}+w\left(\tau_{7}\right) \\
& \leq\left(\tau_{8}-\tau_{7}\right)\left[\left(\tau_{8}-\tau_{7}\right) u\left(\tau_{7}\right)+\left(v\left(\tau_{7}\right)\right)^{p-1}\right]^{1 /(p-1)}+w\left(\tau_{7}\right) \\
& <T\left[T \frac{\varepsilon_{n}^{p-1}}{(2 T)^{p}}+\frac{\varepsilon_{n}^{p-1}}{2(2 T)^{p-1}}\right]^{1 /(p-1)}+\frac{\varepsilon_{n}}{2}<\frac{\varepsilon_{n}}{2}+\frac{\varepsilon_{n}}{2}=\varepsilon_{n} .
\end{aligned}
$$

Consequently, $0<\lambda(t) \leq \varepsilon_{n}, t \in(0, T)_{\mathbb{T}}$, which implies $0<\phi_{m_{1}-1}(\lambda(t)) \leq \phi_{m_{1}-1}\left(\varepsilon_{n}\right), t \in(0, T]_{\mathbb{T}}$. Without loss of generality, one has

$$
\sum_{i=1}^{m_{2}} \psi_{i}\left(\lambda^{\Delta}\left(\xi_{i}\right)\right)-\sum_{j=1}^{m_{1}-1} \phi_{j}\left(\xi_{j}^{\prime}\right) \geq \phi_{m_{1}-1}\left(\varepsilon_{n}\right)>\phi_{m_{1}}\left(\lambda\left(\xi_{m_{1}}^{\prime}\right)\right)=\phi_{m_{1}}(\lambda(T)) .
$$

We have $\phi_{m_{1}}\left(\lambda\left(\xi_{m_{1}}^{\prime}\right)\right)+\sum_{j=1}^{m_{1}-1} \phi_{j}\left(\xi_{j}^{\prime}\right)-\sum_{i=1}^{m_{2}} \psi_{i}\left(\lambda^{\Delta}(\xi i)\right)<0$.

Now we discuss how to construct a lower solution $\alpha(t)$ in (A2) and (A4).

(A7) For each $n \in\{1,2, \ldots\}$, there exist a constant $k_{0}$ and a strictly monotone decreasing sequence $\left\{\rho_{n}\right\}$ with $\lim _{n \rightarrow \infty} \rho_{n}=0$, and $q(t) f\left(t, u(t), u^{\Delta}(t)\right) \geq k_{0}$ for $\left(t, u(t), u^{\Delta}(t)\right) \in$ $\left[1 / 2^{n+1}, T-\left(1 / 2^{n+1}\right)\right]_{\mathbb{T}} \times\left\{u(t) \in C[0, T]_{\mathbb{T}} \cap C^{\Delta}(0, T]_{\mathbb{T}^{\kappa}}: 0<u(t) \leq \rho_{n}\right\} \times(-\infty,+\infty)$.

(A8) There exists a function $\beta(t) \in C[0, T]_{\mathbb{T}} \cap C^{\Delta}(0, T]_{\mathbb{T}^{\kappa}}, \varphi_{p}\left(\beta^{\Delta}(t)\right) \in C^{\nabla}(0, T)_{\mathbb{T}}$ such that $\beta(t) \geq 0$ for $t \in[0, T]_{\mathbb{T}}, \sum_{j=1}^{m_{1}} \phi_{j}\left(\beta\left(\xi_{j}^{\prime}\right)\right)-\sum_{i=1}^{m_{2}} \psi_{i}\left(\beta^{\Delta}\left(\xi_{i}\right)\right)>0,-\left(\varphi_{p}\left(\beta^{\Delta}(t)\right)\right)^{\nabla} \geq$ $q(t) f\left(t, \beta(t), \beta^{\Delta}(t)\right)$ for $t \in(0, T)_{\mathbb{T}}$ and $-\left(\varphi_{p}\left(\beta^{\Delta}(t)\right)\right)^{\nabla} \geq q(t) f\left(1 / 2^{n_{0}+1}, \beta(t), \beta^{\Delta}(t)\right)$ for $t \in\left(0,\left(1 / 2^{n_{0}+1}\right)\right)_{\mathbb{T}}$, and

$$
-\left(\varphi_{p}\left(\beta^{\Delta}(t)\right)\right)^{\nabla} \geq q(t) f\left(T-\frac{1}{2^{n_{0}+1}}, \beta(t), \beta^{\Delta}(t)\right) \text { for } t \in\left(T-\frac{1}{2^{n_{0}+1}}, T\right)_{\mathbb{T}} .
$$


Theorem 3.2. Let $n_{0} \in\{3,4, \ldots\}$ be fixed. If (H1)-(H3), (3.1), and (A7)-(A8) hold, then boundary value problem (1.2) and (1.3) has a solution $u(t) \in C[0, T]_{\mathbb{T}} \cap C^{\Delta}(0, T]_{\mathbb{T}^{k}}$ with $\varphi_{p}\left(u^{\Delta}(t)\right) \in$ $C^{\nabla}(0, T)_{\mathbb{T}}$ and $u(t)>0$ for $t \in(0, T]_{\mathbb{T}}$.

Proof. By Corollary 2.3, we need only to show that conditions (A1), (A2), (A4)-(A6) are satisfied. Without loss of generality, suppose that

$$
\beta(t)>\rho_{n_{0}} \quad \text { for } t \in[0, T]_{\mathbb{T}}, \quad \sum_{j=1}^{m_{1}} \phi_{j}\left(\beta\left(\xi_{j}^{\prime}\right)\right)-\sum_{i=1}^{m_{2}} \psi_{i}\left(\beta^{\Delta}\left(\xi_{i}\right)\right)>\rho_{n_{0}},
$$

by (A7), (A8), and (3.8), we obtain that (A1) and (A5) hold.

From Lemma 3.1 there exists a function $\lambda(t) \in C^{\Delta}[0, T]_{\mathbb{T}}$ satisfying

(i) $\varphi_{p}\left(\lambda^{\Delta}(t)\right) \in C^{\nabla}[0, T]_{\mathbb{T}}, \lambda(t)>0$ for $t \in(0, T]_{\mathbb{T}}$ together with

$$
R_{1}=\max _{t \in[0, T]_{\mathbb{T}}}\left|\left(\varphi_{p}\left(\lambda^{\Delta}(t)\right)\right)^{\nabla}\right|>0
$$

(ii) $\lambda(0)=0, \sum_{j=1}^{m_{1}} \phi_{j}\left(\lambda\left(\xi_{j}^{\prime}\right)\right)-\sum_{i=1}^{m_{2}} \psi_{i}\left(\lambda^{\Delta}\left(\xi_{i}\right)\right)<0$ and $0<\lambda(t) \leq \rho_{n}$ for $t \in(0, T)_{\mathbb{T}}$.

Assume that $m=\min \left\{1,\left(k_{0} / 2 R_{1}\right)^{1 /(p-1)}, \rho_{n_{0}} /|\lambda|\right\}$. Let $\alpha(t)=m \lambda(t)$ for $t \in[0, T]_{\mathbb{T}}$. Then $\alpha(t) \in C[0, T]_{\mathbb{T}} \cap C^{\Delta}(0, T]_{\mathbb{T}^{n}}, \varphi_{p}\left(\alpha^{\Delta}(t)\right) \in C^{\nabla}(0, T)_{\mathbb{T}}, \alpha(0)=0$ with $0<\alpha(t) \leq \lambda(t) \leq \rho_{n}$ for $t \in\left(1 / 2^{n+1}, T\right]_{\mathbb{T}}$. Without loss of generality, we have

$$
\sum_{j=1}^{m_{1}} \phi_{j}\left(\alpha\left(\xi_{j}^{\prime}\right)\right)-\sum_{i=1}^{m_{2}} \psi_{i}\left(\alpha^{\Delta}\left(\xi_{i}\right)\right)<0
$$

For arbitrary

$$
\left(t, u(t), u^{\Delta}(t)\right) \in(0, T]_{\mathbb{T}} \times\{0<u(t) \leq \alpha(t)\} \times\left\{-\infty<u^{\Delta}(t)<+\infty\right\},
$$

there exists $n \in\left\{n_{0}, n_{0}+1, \ldots\right\}$ such that

$$
\left(t, u(t), u^{\Delta}(t)\right) \in\left[\frac{1}{2^{n+1}}, T-\frac{1}{2^{n+1}}\right]_{\mathbb{T}} \times\{0<u(t) \leq \alpha(t)\} \times\left\{-\infty<u^{\Delta}(t)<+\infty\right\} .
$$

We have

$$
\begin{aligned}
q(t) f\left(t, u(t), u^{\Delta}(t)\right)+\left(\varphi_{p}\left(\alpha^{\Delta}(t)\right)\right)^{\nabla} & \geq k_{0}+\left(\varphi_{p}\left(m \lambda^{\Delta}(t)\right)\right)^{\nabla}=k_{0}+m^{p-1}\left(\varphi_{p}\left(\lambda^{\Delta}(t)\right)\right)^{\nabla} \\
& \geq k_{0}-m^{p-1}\left|\left(\varphi_{p}\left(\lambda^{\Delta}(t)\right)\right)^{\nabla}\right| \geq k_{0}-\frac{k_{0}}{2 R_{1}}\left|\left(\varphi_{p}\left(\lambda^{\Delta}(t)\right)\right)^{\nabla}\right| \\
& \geq k_{0}-\frac{k_{0}}{2 R_{1}} \max _{t \in[0, T]}\left|\left(\varphi_{p}\left(\lambda^{\Delta}(t)\right)\right)^{\nabla}\right|=\frac{k_{0}}{2}>0 .
\end{aligned}
$$


Thus (A4) holds and (A2) is also true if $u(t)=\alpha(t)$. Further, since $\alpha(T) \leq \sup _{[0, T]}|\alpha(t)|=$ $m \sup _{t \in[0, T]_{T}}|\lambda(t)| \leq \rho_{n_{0}}$, we have $\beta(T) \geq \rho_{n_{0}} \geq \alpha(T)$, then (A6) is fulfilled. By Corollary 2.3, the boundary value problem (1.2) and (1.3) has a solution $u(t) \in C[0, T]_{\mathbb{T}} \cap C^{\Delta}(0, T]_{\mathbb{T}^{\kappa}}, \varphi_{p}\left(u^{\Delta}(t)\right) \in$ $C^{\nabla}(0, T)_{\mathbb{T}}$ with $u(t) \geq 0$ for $t \in(0, T]_{\mathbb{T}}$.

According to Theorem 2.1, it is difficult for us to discuss examples in constructing $\beta$ in (A8). The following theorem removes (A8) and replaces it with an easy verified condition.

Theorem 3.3. Let $n_{0} \in\{1,2, \ldots\}$ be fixed. If (H1)-(H3), (A1), and (A2) hold, in addition suppose that the following conditions are satisfied:

$$
\begin{gathered}
M_{1}>0, \quad M_{2}>\max \left\{\sup _{t \in[0, T]_{\mathbb{T}}} \alpha(t), \rho_{n_{0}}\right\}, \quad \text { here } M_{1}, M_{2} \text { are constants, } \\
q(t) f\left(t, M_{1} t+M_{2}, M_{1}\right) \leq 0 \text { for } t \in(0, T)_{\mathbb{T}}, \\
q(t) f\left(\frac{1}{2^{n_{0}+1}}, M_{1} t+M_{2}, M_{1}\right) \leq 0 \text { for } t \in\left(0, \frac{1}{2^{n_{0}+1}}\right)_{\mathbb{T}}, \\
q(t) f\left(\frac{1}{2^{n_{0}+1}}, M_{1} t+M_{2}, M_{1}\right) \leq 0 \text { for } t \in\left(T-\frac{1}{2^{n_{0}+1}}, T\right)_{\mathbb{T}^{\prime}} \\
\sum_{j=1}^{m_{1}} \phi_{j}\left(M_{1} \xi_{j}^{\prime}+M_{2}\right)-\sum_{i=1}^{m_{2}} \psi_{i}\left(M_{1}\right)>0 .
\end{gathered}
$$

Then boundary value problem (1.2) and (1.3) has a solution $u(t) \in C[0, T]_{\mathbb{T}} \cap C^{\Delta}(0, T]_{\mathbb{T}^{\kappa}}$ with $\varphi_{p}\left(u^{\Delta}(t)\right) \in C^{\nabla}(0, T)_{\mathbb{T}}$ and $u(t)>0$ for $t \in(0, T]_{\mathbb{T}}$.

Proof. Denote $\beta(t)=M_{1} t+M_{2}$ for $t \in[0, T]_{\mathbb{T}}$, then $\beta(t) \in C[0, T]_{\mathbb{T}} \cap C^{\Delta}(0, T]_{\mathbb{T}^{x}}, \varphi_{p}\left(\beta^{\Delta}(t)\right) \in$ $C^{\nabla}(0, T)_{\mathbb{T}}$ together with $\beta(t) \geq \alpha(t)$ and $\beta(t) \geq \rho_{n_{0}}$ for $t \in[0, T]_{\mathbb{T}}, \sum_{j=1}^{m_{1}} \psi_{i}\left(\beta\left(\xi_{j}^{\prime}\right)\right)-$ $\sum_{i=1}^{m_{2}} \psi_{i}\left(\beta^{\Delta}\left(\xi_{i}\right)\right)>0$, with

$$
\begin{aligned}
& -\left(\varphi_{p}\left(\beta^{\Delta}(t)\right)\right)^{\nabla} \geq q(t) f\left(t, \beta(t), \beta^{\Delta}(t)\right) \quad \text { for } t \in(0, T)_{\mathbb{T}} \\
& -\left(\varphi_{p}\left(\beta^{\Delta}(t)\right)\right)^{\nabla} \geq q(t) f\left(\frac{1}{2^{n_{0}+1}}, \beta(t), \beta^{\Delta}(t)\right) \quad \text { for } t \in\left(T-\frac{1}{2^{n_{0}+1}}, \frac{1}{2^{n_{0}+1}}\right)_{\mathbb{T}}, \\
& -\left(\varphi_{p}\left(\beta^{\Delta}(t)\right)\right)^{\nabla} \geq q(t) f\left(\frac{1}{2^{n_{0}+1}}, \beta(t), \beta^{\Delta}(t)\right) \text { for } t \in\left(0, \frac{1}{2^{n_{0}+1}}\right)_{\mathbb{T}},
\end{aligned}
$$

then (A3) holds. By Theorem 2.1 the result holds.

From Theorems 2.1 and 3.2 we have the following theorem.

Theorem 3.4. Let $n_{0} \in\{1,2, \ldots\}$ be fixed. If (H1)-(H3), (3.1), and (A7) hold, in addition suppose that there exist constants $M_{1}, M_{2}>0$ such that (3.15) and (3.16) are true. Then the problem (1.2) and (1.3) has a solution $u(t) \in C[0, T]_{\mathbb{T}} \cap C^{\Delta}(0, T]_{\mathbb{T}^{\kappa}}$ with $\varphi_{p}\left(u^{\Delta}(t)\right) \in C^{\nabla}(0, T)_{\mathbb{T}}$ and $u(t)>0$ for $t \in(0, T]_{\mathbb{T}}$. 
Proof. Without loss of generality, suppose that $\rho_{n_{0}}<M_{2}$, by (A7), (A1) holds and

$$
M_{2}>\rho_{n_{0}}>\rho_{n_{0}+1}>\cdots, \quad \lim _{n \rightarrow \infty} \rho_{n}=0
$$

By the similar way as the proof of Theorem 3.2, there exists a function $\alpha(t) \in C[0, T]_{\mathbb{T}} \cap$ $C^{\Delta}(0, T]_{\mathbb{T}^{\kappa}}, \varphi_{p}\left(\alpha^{\Delta}(t)\right) \in C^{\nabla}(0, T)_{\mathbb{T}}$ with $\alpha(0)=0, \sum_{j=1}^{m_{1}} \phi_{j}\left(\alpha\left(\xi_{j}^{\prime}\right)\right)-\sum_{i=1}^{m_{2}} \psi_{i}\left(\alpha^{\Delta}\left(\xi_{i}\right)\right)<0, \alpha(t)>$ 0 for $t \in(0, T]_{\mathbb{T}}$, such that $-\left(\varphi_{p}\left(\alpha^{\Delta}(t)\right)\right)^{\nabla} \leq q(t) f\left(t, \alpha(t), \alpha^{\Delta}(t)\right)$ for $t \in(0, T)_{\mathbb{T}}$ and $\alpha(t) \leq$ $\sup _{t \in[0, T]_{\mathbb{T}}}|\alpha(t)| \leq \rho_{n_{0}}$. By this together with (3.18), we have $M_{2}>\max \left\{\sup _{t \in[0, T]_{\mathbb{T}}} \alpha(t), \rho_{n_{0}}\right\}$. Thus all the conditions of Theorem 3.3 are fulfilled.

\section{An Example}

In this section, we present an example to illustrate our results.

Let $\mathbb{T}=\{0\} \cup\left\{\left(\frac{1}{2}\right)^{\mathbb{N}}\right\} \cup\left[\frac{1}{2,1}\right]$. Consider the following boundary value problem:

$$
\begin{gathered}
-\left(\left|u^{\Delta}(t)\right|^{2} u^{\Delta}(t)\right)^{\nabla}=q(t) f\left(t, u(t), u^{\Delta}(t)\right) \quad \text { for } t \in(0,1)_{\mathbb{T}^{\prime}} \\
u(0)=0, \quad u(1)+\frac{1}{5} u\left(\frac{1}{8}\right)-\frac{1}{5} u^{\Delta}\left(\frac{1}{8}\right)-\frac{1}{10} u^{\Delta}\left(\frac{1}{4}\right)-\frac{1}{5} u^{\Delta}\left(\frac{3}{4}\right)-\frac{1}{10} u^{\Delta}(1)=0 .
\end{gathered}
$$

It is obvious that $T=1, p=4, \varphi_{1}(x)=\psi_{1}(x)=\left(\frac{1}{5}\right) x, \psi_{2}(x)=\left(\frac{1}{10}\right) x, \psi_{3}(x)=\left(\frac{1}{5}\right) x$, and $\psi_{4}(x)=x$. Denote $q(t)=t^{4}+4$ and $f\left(t, u(t), u^{\Delta}(t)\right)=t / u^{7}(t)+u^{8}(t)+\left(t u^{\Delta}(t)\right)^{2}-\lambda^{2}$, here $\lambda^{2} \geq 10$ is constant. Let $n_{0} \in\{1,2, \ldots\}, \rho_{n}=\left(1 / 2^{n+1}\left(\lambda^{2}+a_{1}\right)\right)^{1 / 7}$, and $k_{0}=a_{1}>0$ is a constant, we have $\rho_{n_{0}} \leq 1$. Note that (H1)-(H3) and (3.1) hold. For $n \in\{1,2, \ldots\}, t \in\left[1 / 2^{n+1}, 1-\left(1 / 2^{n+1}\right)\right]_{\mathbb{T}}$, and $0<u \leq \rho_{n}$, we have

$$
q(t) f\left(t, u, u^{\Delta}\right) \geq\left(t^{4}+4\right)\left(\frac{1}{2^{n+1} \rho_{n}^{7}}-\lambda^{2}\right) \geq\left(t^{4}+4\right)\left(\lambda^{2}+a_{1}-\lambda^{2}\right)>a_{1}
$$

which implies that (A7) is satisfied.

Now we show that $(\mathrm{A} 8)$ holds with $\beta(t)=t^{1 / 7}$.

Notice that if $t \in(1 / 2,1]_{\mathbb{T}}$, then $\beta^{\Delta}(t)=\beta^{\prime}(t)=(1 / 7) t^{-6 / 7}$,

$$
\begin{gathered}
\left|\beta^{\Delta}(t)\right|^{2} \beta^{\Delta}(t)=\frac{1}{343} t^{-18 / 7}, \quad\left(\left|\beta^{\Delta}(t)\right|^{2} \beta^{\Delta}(t)\right)^{\nabla}=-\frac{18}{2401} t^{-25 / 7} \leq 0 . \\
\text { If } t=1 / 2 \text {, then } \beta^{\Delta}(t)=(1 / 7) t^{-6 / 7} \text { and }\left|\beta^{\Delta}(t)\right|^{2} \beta^{\Delta}(t)=(1 / 343) t^{-18 / 7}, \\
\left(\left|\beta^{\Delta}(t)\right|^{2} \beta^{\Delta}(t)\right)^{\nabla}=\frac{2}{343}\left(\frac{1}{2}\right)^{-18 / 7}-\frac{2}{343}\left(\frac{1}{4}\right)^{-18 / 7} \approx-0.1714 \leq 0 .
\end{gathered}
$$




$$
\begin{gathered}
\text { If } t=1 / 2^{n}(n=2,3, \ldots) \text {, then } \sigma(t)=2 t, \rho(t)=t / 2, \mu(t)=t, v(t)=t / 2 \text {, we have } \\
\qquad \beta^{\Delta}(t)=\frac{1}{t}\left[(2 t)^{1 / 7}-t^{1 / 7}\right], \quad\left|\beta^{\Delta}(t)\right|^{2} \beta^{\Delta}(t)=\frac{1}{t^{3}}\left[(2 t)^{1 / 7}-t^{1 / 7}\right]^{3},
\end{gathered}
$$

by induction, one gets

$$
\left(\left|\beta^{\Delta}(t)\right|^{2} \beta^{\Delta}(t)\right)^{\nabla}=2^{4 n+1}\left[\left(\frac{1}{2^{n-1}}\right)^{1 / 7}-\left(\frac{1}{2^{n}}\right)^{1 / 7}\right]^{3}-2^{4 n+4}\left[\left(\frac{1}{2^{n}}\right)^{1 / 7}-\left(\frac{1}{2^{n+1}}\right)^{1 / 7}\right]^{3} \leq 0
$$

Thus, for $t \in(0,1]_{\mathbb{T}}$, we have

$$
\begin{aligned}
& \left(\left|\beta^{\Delta}(t)\right|^{2} \beta^{\Delta}(t)\right)^{\nabla}+q(t) f\left(t, \beta(t), \beta^{\Delta}(t)\right) \\
& \quad \leq\left\{\begin{array}{l}
\left(t^{4}+4\right)\left(\frac{t}{t}+t^{8 / 7}+\left(\frac{1}{7} t^{1 / 7}\right)^{2}-\lambda^{2}\right), \\
\left(t^{4}+4\right)\left(\frac{t}{t}+t^{8 / 7}+\left((2 t)^{1 / 7}-t^{1 / 7}\right)^{2}-\lambda^{2}\right), \quad t \in\left\{\frac{1}{2^{n}}, n=2,3, \ldots,\right\},
\end{array}\right. \\
& \leq\left(t^{4}+4\right)\left(10-\lambda^{2}\right) \leq 0, \\
& \left(\left|\beta^{\Delta}(t)\right|^{2} \beta^{\Delta}(t)\right)^{\nabla}+q(t) f\left(\frac{1}{2^{n_{0}+1}}, \beta(t), \beta^{\Delta}(t)\right) \\
& \leq\left(t^{4}+4\right)\left(\frac{1}{2^{n_{0}+1} t}+\left(t^{1 / 7}\right)^{8}+\left((2 t)^{1 / 7}-t^{1 / 7}\right)^{2}-\lambda^{2}\right) \\
& \leq\left(t^{4}+4\right)\left(10-\lambda^{2}\right) \leq \text { for } t \in\left(0, \frac{1}{2^{n_{0}+1}}\right)_{\mathbb{T}} .
\end{aligned}
$$

In addition

$$
\beta(1)+\varphi_{1}\left(\beta\left(\frac{1}{8}\right)\right)-\psi_{1}\left(\beta^{\Delta}\left(\frac{1}{8}\right)\right)-\psi_{2}\left(\beta\left(\frac{1}{4}\right)\right)-\psi_{3}\left(\beta^{\Delta}\left(\frac{3}{4}\right)\right)-\psi_{4}(\beta(1))>0 .
$$

Hence, all conditions of Theorem 3.2 are satisfied. As a result, the problem (4.1) has a positive solution.

\section{Acknowledgment}

This paper is supported by XZIT under Grant no. XKY2008311. 


\section{References}

[1] S. Hilger, Ein Maßkettenkalkül mit Anwendung auf Zentrumsmannigfaltigkeiten, Ph. D. thesis, Universität Würzburg, Würzburg, Germany, 1988.

[2] M. Bohner and A. Peterson, Eds., Advances in Dynamic Equations on Time Scales, Birkhäuser, Boston, Mass, USA, 2003.

[3] M. Bohner and A. Peterson, Dynamic Equations on Time Scales: An Introduction with Applications, Birkhäuser, Boston, Mass, USA, 2001.

[4] M.-Q. Feng, X.-G. Li, and W.-G. Ge, "Triple positive solutions of fourth-order four-point boundary value problems of $p$-Laplacian dynamic equations on time scales," Advances in Difference Equations, vol. 2008, Article ID 496078, 9 pages, 2008.

[5] Y.-H. Su, "Multiple positive pseudo-symmetric solutions of $p$-Laplacian dynamic equations on time scales," Mathematical and Computer Modelling, vol. 49, no. 7-8, pp. 1664-1681, 2009.

[6] Y.-H. Su and W. T. Li, "Existence of positive solutions to a singular $p$-Laplacian dynamic equations with sign changing nonlinearity," Acta Mathematica Sinica, Chinese Series, vol. 28, pp. 51-60, 2008.

[7] Y.-H. Su, X.-H. Yuan, and X. X. Yan, "Existence of solution to a three-point BVPs for $p$-Laplacian dynamic equations on time scales," Journal of Lanzhou University, Natural Sciences, vol. 44, pp. 112116, 2008.

[8] Y.-H. Su and W.-T. Li, “Triple positive solutions of $m$-point BVPs for $p$-Laplacian dynamic equations on time scales," Nonlinear Analysis: Theory, Methods \& Applications, vol. 69, no. 11, pp. 3811-3820, 2008.

[9] Y.-H. Su, W.-T. Li, and H.-R. Sun, "Triple positive pseudo-symmetric solutions of three-point BVPs for $p$-Laplacian dynamic equations on time scales," Nonlinear Analysis: Theory, Methods E Applications, vol. 68, no. 6, pp. 1442-1452, 2008.

[10] H.-R. Sun and W.-T. Li, "Positive solutions of $p$-Laplacian $m$-point boundary value problems on time scales," Taiwanese Journal of Mathematics, vol. 12, no. 1, pp. 105-127, 2008.

[11] H.-R. Sun and W.-T. Li, "Existence theory for positive solutions to one-dimensional $p$-Laplacian boundary value problems on time scales," Journal of Differential Equations, vol. 240, no. 2, pp. 217248, 2007.

[12] C. Song, "Eigenvalue problems for $p$-Laplacian functional dynamic equations on time scales," Advances in Difference Equations, vol. 2008, Article ID 879140, 9 pages, 2008.

[13] V. Spedding, "Taming nature's numbers," New Scientist, vol. 179, no. 2404, pp. 28-31, 2003.

[14] F. M. Atici, D. C. Biles, and A. Lebedinsky, "An application of time scales to economics," Mathematical and Computer Modelling, vol. 43, no. 7-8, pp. 718-726, 2006.

[15] Q. Sheng, M. Fadag, J. Henderson, and J. M. Davis, "An exploration of combined dynamic derivatives on time scales and their applications," Nonlinear Analysis: Real World Applications, vol. 7, no. 3, pp. 395-413, 2006.

[16] D. M. Thomas, L. Vandemuelebroeke, and K. Yamaguchi, "A mathematical evolution model for phytoremediation of metals," Discrete and Continuous Dynamical Systems. Series B, vol. 5, no. 2, pp. 411-422, 2005.

[17] Y.-H. Su, W.-T. Li, and H.-R. Sun, "Positive solutions of singular $p$-Laplacian BVPs with sign changing nonlinearity on time scales," Mathematical and Computer Modelling, vol. 48, no. 5-6, pp. 845-858, 2008.

[18] Y.-H. Su and W. T. Li, "Existence of positive solutions to a singular $p$-Laplacian dynamic equations with sign changing nonlinearity," Acta Mathematica Sinica, Chinese Series, vol. 52, pp. 181-196, 2009.

[19] Y.-H. Su, W.-T. Li, and H.-R. Sun, "Positive solutions of singular $p$-Laplacian dynamic equations with sign changing nonlinearity," Applied Mathematics and Computation, vol. 200, no. 1, pp. 352-368, 2008.

[20] Q. Wei, Y.-H. Su, S. Li, and X.-X. Yan, "Existence of positive solutions to a singular $p$-Laplacian general dirichlet BVPs with sign changing nonlinearity," Abstract and Applied Analysis, vol. 2009, Article ID 512402, 21 pages, 2009.

[21] B. Jain and A. D. Sheng, "An exploration of the approximation of derivative functions via finite differences," Rose-Hulman Undergraduate Mathematics Journal, vol. 8, no. 2, pp. 1-19, 2007.

[22] J. W. Rogers, Jr. and Q. Sheng, "Notes on the diamond- $\alpha$ dynamic derivative on time scales," Journal of Mathematical Analysis and Applications, vol. 326, no. 1, pp. 228-241, 2007.

[23] Q. Sheng, "A view of dynamic derivatives on time scales from approximations," Journal of Difference Equations and Applications, vol. 11, no. 1, pp. 63-81, 2005.

[24] R. P. Agarwal, H. Lü, and D. O’Regan, "Existence theorems for the one-dimensional singular $p$ Laplacian equation with sign changing nonlinearities," Applied Mathematics and Computation, vol. 143, no. 1, pp. 15-38, 2003. 
[25] D. O'Regan, "Upper and lower solutions for singular problems arising in the theory of membrane response of a spherical cap," Nonlinear Analysis: Theory, Methods \& Applications, vol. 47, no. 2, pp. 1163-1174, 2001.

[26] H. Lü, D. O’Regan, and R. P. Agarwal, "Existence theorems for the one-dimensional singular $p$ Laplacian equation with a nonlinear boundary condition," Journal of Computational and Applied Mathematics, vol. 182, no. 1, pp. 188-210, 2005.

[27] H. Lü, D. O’Regan, and R. P. Agarwal, "Positive solutions for singular $p$-Laplacian equations with sign changing nonlinearities using inequality theory," Applied Mathematics and Computation, vol. 165, no. 3, pp. 587-597, 2005.

[28] H. Lü, D. O’Regan, and R. P. Agarwal, “Upper and lower solutions for the singular $p$-Laplacian with sign changing nonlinearities and nonlinear boundary data," Journal of Computational and Applied Mathematics, vol. 181, no. 2, pp. 442-466, 2005.

[29] V. Lakshmikantham, S. Sivasundaram, and B. Kaymakcalan, Dynamic Systems on Measure Chains, vol. 370 of Mathematics and Its Applications, Kluwer Academic Publishers, Dordrecht, The Netherlands, 1996.

[30] R. P. Agarwal, M. Bohner, and P. Řehák, "Half-linear dynamic equations," in Nonlinear Analysis and Applications: to V. Lakshmikantham on His 80th Birthday. Vol. 1, 2, pp. 1-57, Kluwer Academic Publishers, Dordrecht, The Netherlands, 2003. 